\title{
In-plane Dynamic Crushing of a Novel Bio-inspired Re-entrant Honeycomb With Negative Poisson's Ratio
}

\author{
Yonghui Wang \\ Jiangsu University of Science and Technology \\ Qiang He (D 649668684@qq.com) \\ Jiangsu University of Science and Technology \\ Yu Chen \\ Jiangsu Institute of Technology \\ Hang Gu \\ Jiangsu University of Science and Technology \\ Honggen Zhou \\ Jiangsu University of Science and Technology
}

\section{Original Article}

Keywords: bio-inspired re-entrant honeycomb (BRH), numerical simulation, crushing behavior, deformation mechanism, energy absorption capacity

Posted Date: February 9th, 2021

DOI: https://doi.org/10.21203/rs.3.rs-195463/v1

License: (c) (i) This work is licensed under a Creative Commons Attribution 4.0 International License. Read Full License 


\title{
In-plane dynamic crushing of a novel bio-inspired re- entrant honeycomb with negative Poisson's ratio
}

\begin{abstract}
Yonghui Wang ${ }^{1}$, Qiang He ${ }^{1, *}$, Yu Chen ${ }^{2}$, Hang Gu ${ }^{1}$, Honggen Zhou ${ }^{1}$
Abstract: In order to seek higher crashworthiness and energy absorption capacity, based on biological inspiration, a novel bio-inspired re-entrant honeycomb (BRH) structure with negative Poisson's ratio is designed by selecting lotus leaf vein as biological prototype. The numerical simulation model is established by the nonlinear dynamics software ABAQUS and further compared with the available reference results to verify the feasibility. The dynamic compression behavior and energy absorption capacity of two types of BRH (BRH-I and BRH-II) are firstly compared with conventional re-entrant honeycomb $(\mathrm{RH})$. The simulation results show that BRH have better mechanical properties and energy absorption characteristics. Then, the crushing behavior of BRH-II under different impact velocities are systematically studied. Three typical deformation modes of BRH-II are observed through the analysis of deformation profile. The quasi-static plateau stress is closely related to the cellular structure. Based on onedimensional shock theory, the empirical equations of dynamic plateau stress for BRH-II with different relative densities are given by using least-square fitting. In addition, the effects of impact velocity and relative density on plateau stress and energy absorption behavior are also studied. The results show that the energy absorption capacity of BRH-II is increased nearly six times compared with RH at the same impact velocity.
\end{abstract}

Keywords: bio-inspired re-entrant honeycomb (BRH); numerical simulation; crushing behavior; deformation mechanism; energy absorption capacity;

\section{Introduction}

Due to the excellent specific strength, specific stiffness and energy absorption characteristics [1], honeycomb structure has been widely used in aerospace [2-3], broadside protection structure and other fields. Therefore, a lot of research has been done on its in-plane and out-of-plane compression characteristics [4-5]. Hu et al. [6] carried out a finite element simulation on the inplane compression of a polycarbonate circular honeycomb, and characterized the uneven deformation and its evolution in the honeycomb block. Cricrì et al. [7] studied the compression behavior of conventional hexagonal honeycombs based on experiments and numerical simulations. Yang et al. [8] proposed a quasi-hexagonal honeycomb structure based on the finite element method to study its mechanical properties under uniform compression outside the quasi-static plane. Liang et al. [9] studied the collapse of a square honeycomb sandwich panel and revealed some mechanical properties of the square honeycomb sandwich structure.

With the development of negative Poisson's ratio materials, honeycomb structures with negative Poisson's ratio performance have also been continuously researched and discovered. The honeycomb with negative Poisson has better mechanical properties than conventional honeycomb structures due to the characteristics of horizontal shrinkage, shear modulus [10], quasi-static and dynamic impact resistance [11-12] under lateral load. After Lakes [13] prepared polyurethane foam with Poisson's ratio of -0.7 , scholars successively designed various honeycomb structures with NPR. Hou et al. [14] proposed a new isotropic negative Poisson's ratio composite structure. Wang et al. [15] introduced peanut-like holes into the solid block matrix and designed a novel negative Poisson's ratio structure. Gao et al. [16] designed a three-dimensional double- $\mathrm{V}$ honeycomb intersected by a negative Poisson's ratio two-dimensional double-V structure. Qin et al. [17] designed a series of 
metamaterial structures with arbitrary NPR (ratio range from -0.3 to -6.0). Xiao et al. [18] studied in detail the effect of negative Poisson's ratio (NPR) on the shrinkage deformation and compressive stress of metal ARH under low, medium, and high-velocity compression, and revealed an I+V hybrid deformation mode. Hu et al. [19] conducted a theoretical analysis on the NPR effect and crushing stress of reentrant hexagonal honeycombs, and the results showed that the crushing stress increased significantly with the increase of crushing velocity. Tan et al. [20] proposed hierarchical honeycomb structures and studied their crashworthiness under quasi-static in-plane compression. The results show that these two layered honeycombs have better mechanical and energy absorption properties. Yang et al. [21] studied the in-plane crushing behavior and energy absorption capacity of the established petal-shaped honeycomb (PSH) 3D finite element model under different impact velocities. Three typical deformation modes, namely quasi-static, transition and dynamic mode were observed and summarizes the destruction mode diagram.

With the increasing demand for the crashworthiness and energy absorption capacity of honeycomb structure, how to design a negative Poisson's ratio honeycomb structure with higher crashworthiness and energy absorption capacity remains a challenge. As we all know, bionic design is a very interesting and effective design method that can improve the mechanical properties of regular structure. Therefore, many scholars are inspired by the biological structures and designed many new types of crashworthy structures [22-32]. Zou et al. [24] designed a bionic tube consisting of a bionic node, a bionic unit and an inner tube, the results show that the gradient distribution of the vascular bundle and the node can improve energy absorption. Song et al. [25] was inspired by the thickness gradient and the internode distance along the growth direction of the bamboo proposed a variable thickness structure (VTS) energy absorbing structure. The study found that some VTS have better crash resistance than round tubes.

The special structure of lotus leaf vein is conducive to the reasonable transmission of load, so that it can bear strong load. Therefore, lotus leaf vein can be regarded as a potential bionic design object. So far, no relevant research has introduced this structural feature into the design of honeycomb structures. In this paper, a novel bio-inspired re-entrant honeycomb (BRH) with negative Poisson's ratio is proposed inspired by the branching characteristics of lotus leaf veins. Based on finite element simulation, the crashworthiness and energy absorption capacity of BRH under the same crushing load are quantitatively compared with the conventional re-entrant honeycomb $(\mathrm{RH})$ with negative Poisson's ratio. Then the in-plane crushing behavior and energy absorption characteristics of BRH-II under different impact velocities are systematically studied, including the typical crushing modes, plateau stress, shrinkage deformation and energy absorption characteristics.

\section{Geometric model of BRH}

\subsection{Geometry of BRH}

The strong carrying capacity of lotus leaf is due to the special distribution of its vein, which realizes the reasonable transmission of the load. Therefore, the geometric characteristics of lotus leaf vein branch may open up a new design idea for the design of honeycomb structure to improve their mechanical properties by introducing such structural features. Fig. 1 shows the design process inspired by the branch of lotus leaf vein. It can be seen from Fig. 1(a) that the geometric characteristics of lotus leaf vein are mainly reflected in the main-veins and branch-veins. Combining this characteristic with unit cell of conventional re-entrant honeycomb (RH), two new representative 
unit cell can be obtained. And then two types of bio-inspired re-entrant honeycombs (BRH-I, BRHII) are depicted in Fig. 1(b).

Fig. 2 depicted the geometrical characteristic of representative unit cell of BRH in great detail. The height $H_{0}$ of the representative unit cell, the angle $\alpha$ between the hypotenuse and the horizontal side, and the branch angle $\beta$ are $8 \mathrm{~mm}, 53.15^{\circ}, 73.71^{\circ}$, respectively. The side lengths $H, L$ and $L_{0}$ are $12 \mathrm{~mm}, 5 \mathrm{~mm}$, and $5 \mathrm{~mm}$ respectively. Therefore, the length $L_{1}$ and height $L_{2}$ of BRH are respectively equal to $76 \mathrm{~mm}$ and $48 \mathrm{~mm}$. As shown in Fig. 2 (a) and (b), the red and blue dots in the structural units of BRH-I and BRH-II are divided into midpoints and quarter points representing the corresponding edges. RH consists of 30 unit-cells in six rows and five columns, which is exactly the same as that in Ref. [18].

Based on the previous theoretical analysis, the relative densities of BRH-I and BRH-II can be calculated by:

$$
\begin{gathered}
\bar{\rho}_{\mathrm{I}}=\frac{5\left(H_{0}+3 L_{0}\right)}{3 H_{0}} \frac{t_{0}}{L_{0}} \\
\overline{\rho_{\mathrm{II}}}=\frac{5\left(H_{0}+4 L_{0}\right)}{3 H_{0}} \frac{t_{0}}{L_{0}}
\end{gathered}
$$

where $\overline{\rho_{\mathrm{I}}}, \overline{\rho_{\mathrm{II}}}$ are respectively the relative densities of BRH-I and BRH-II. As shown in Eqs. (1) and (2), although their relative densities can be adjusted by changing the structural parameters of BRH-I and BRH-II, changing the thickness may be the simplest and most effective method. When studying the influence of relative density on plateau stress and energy absorption, this method is used to adjust its relative density.

\subsection{Crashworthiness indicators}

In order to evaluate the crashworthiness of $\mathrm{BRH}$, it is very important to define the crashworthiness index. In this study, the plateau stress and specific energy absorption are two main indicators to measure the crashworthiness of all honeycomb structures. The plateau stress is the average stress between the yield strain of the structure and the densification strain. The calculation formula is as follows:

$$
\bar{\sigma}_{p}=\frac{1}{\varepsilon_{d}-\varepsilon_{y}} \int_{\varepsilon_{y}}^{\varepsilon_{d}} \sigma(\varepsilon) d \varepsilon
$$

where $\varepsilon_{y}$ is the yield strain of the structure, $\varepsilon_{d}$ is the densification strain. EA is the total energy absorbed by the structure during the impact, it can be given as:

$$
E A(x)=\int_{0}^{s} F(x) d x
$$

where $F(x)$ is the crushing force and $s$ is the total compression displacement. SEA is the energy absorption per unit mass, which can reflect energy absorption capacity of the structure. It can be calculated by:

$$
S E A=\frac{E A(x)}{M}
$$

where $M$ is the total mass of the structure, and these crashworthiness indexes can be obtained from the force and displacement curves. Obviously, the larger the $S E A$, the better energy absorption characteristics of the structure. In addition, a large plateau stress means more energy can be absorbed and the compressive stress is relatively stable. Therefore, generally the greater the plateau stress, the better the crashworthiness of the structure. 


\section{Finite element model}

\subsection{Modeling method}

In order to study the crashworthiness and energy absorption capacity of BRH, the nonlinear dynamics software Abaqus is used to simulate the in-plane dynamic compression process of BRH. Fig. 3 shows the finite element model of BRH under in-plane impact. The finite element model includes three parts, namely the top plate, the bottom plate and the BRH. In the simulation process, BRH is placed between the top plate and the bottom plate. The bottom plate is set as a fixed constraint. The top plate compresses the honeycomb structure downward at a constant velocity of $1 \mathrm{~m} / \mathrm{s}-200 \mathrm{~m} / \mathrm{s}$. The material constitutive of BRH adopts an ideal elastoplastic model. Its material parameters are density $2700 \mathrm{~kg} / \mathrm{m}^{3}$, Young's modulus $68 \mathrm{GPa}$, Poisson's ratio 0.3 , yield stress $255 \mathrm{MPa}$ [18], and the top and bottom plates are set as rigid bodies. BRH, top plate and bottom plate are all modeled by four-node linear quadrilateral shell elements, and five thickness integration points are used to ensure convergence. In addition, the contact between the BRH and the top and bottom plates is set to a general contact algorithm with a friction coefficient of 0.2 and a hard contact to prevent penetration and relative sliding between the structures. Finally, the convergence analysis is carried out through simulation. The mesh size is set to $0.75 \mathrm{~mm} \times 0.75 \mathrm{~mm}$ after a trade-off between accuracy and computational cost.

\subsection{Validation of FE model}

In order to verify the validity of the finite element model in this study, the compressive stress and deformation modes of conventional RH under quasi-static, low-velocity, medium-velocity and high-velocity impact are compared and analyzed with results in Ref. [18]. As shown in Fig. 4 (a), the nominal stress-strain curve and the energy absorption value per unit volume of the present simulation model are in good agreement with Ref. [18]. The difference lies in the peak value and fluctuation of the stress-strain curve. At the same time, the calculated plateau stress of the finite element simulation is only $1.4 \%$ lower than that in Ref. [18], which further demonstrates that the slight difference of the stress-strain curve will not greatly affect the total absorption energy and SEA. As shown in Fig. 4 (b), during the dynamic compression process, the RH first appeared a "Y"shaped local shear zone, and then formed an "I"-shaped shear in the middle area, and finally RH was compacted. From the perspective of the entire compression process, the negative Poisson's ratio effect of RH is due to its shrinkage deformation.

Fig. 5 shows the comparison of stress-strain curves at the impact end. Under low, medium, and high-velocity impact, the changing trend of stress-strain curve is consistent with that in Ref. [18] and the deviations of plateau stress and densification strain are all within the allowable range. As the corresponding deformation modes shown in Fig. 6, the finite element simulation of NPRH under medium and high-speed impact is very consistent with the reference results, but there are differences under low-velocity compression. Through comparison, it is found that the RH of Ref. [33] has a similar symmetrical deformation mode to the simulation results under low-speed compression. This asymmetrical deformation mode in Ref. [18] may be caused by the unfavorable sliding between the plate and the honeycomb during low-velocity compression. Comprehensive analysis shows that the present FE model method is accurate and effective, so it can be used to establish BRH finite element model. 


\section{Comparative analysis of three honeycomb structures}

In order to illustrate the excellent crashworthiness of BRH, the dynamic response curves of BRH and conventional RH under impact velocity of $1 \mathrm{~m} / \mathrm{s}, 50 \mathrm{~m} / \mathrm{s}$, and $100 \mathrm{~m} / \mathrm{s}$ are compared and analyzed. Fig. 7 shows the stress-strain curves of conventional RH, BRH-I, BRH-II under different impact velocities. The crushing process of the three honeycomb structures basically presents three stages: the initial linear elastic stage, the stable crushing stage, and the densification stage. From Fig. 7 (a) and (b), it can be seen that the plateau stresses of BRH-I and BRH-II at impact velocity of $1 \mathrm{~m} / \mathrm{s}$ and $50 \mathrm{~m} / \mathrm{s}$ are significantly greater than that of conventional RH. However, the plateau stresses of the three honeycomb structures under $100 \mathrm{~m} / \mathrm{s}$ impact velocity are almost the same in Fig. 7(c), indicating that the impact velocity has a great influence on the energy absorption of honeycomb structure. It is generally believed that higher plateau stress means better crashworthiness, so the crashworthiness of BRH-I and BRH-II is better than conventional RH at impact velocities of $1 \mathrm{~m} / \mathrm{s}$ and $50 \mathrm{~m} / \mathrm{s}$. Meanwhile, the initial stress peak increases with the increase of impact velocity. This is because the greater the impact velocity, the greater the effect of inertia. As shown in Fig. 7 (a), the stress of BRH-I in the early stage of stable crushing is greater than that of BRH-II. With the continuous compression of the rigid plate, BRH-II shows stronger energy absorption ability. However, the difference of stress-strain curves between BRH-I and BRH-II decreases with the increase of impact velocity.

The deformation mechanism has an important influence on its energy absorption performance. Thus, the deformation modes of these three honeycombs under different impact velocities are compared. Fig. 8 (a) shows the typical deformation modes of the three honeycomb structures under low-velocity impact. The conventional RH presents a "Y" crushing mode and a "V+X" mixed crushing mode. The formation of this local shear band is due to the free edge effect on the side of honeycomb. The degree of freedom of the cells near the central area of the honeycomb structure is smaller than that of the cells near the sides and the cross-sectional constraints may change during the crushing process. Therefore, the deformation of cells along the cross section are different from each other, resulting in the formation of local shear bands. The generation of this local deformation zone will directly affect the effective crushing force. While for the BRH-I and BRH-II, only a single "X" local shear zone appeared in the early stage, and then no obvious local shear zone is observed and the cells in the entire red box may be seriously deformed. There is no doubt that the uniform plastic deformation of all the cells within the red box will enhance its energy absorption ability.

For BRH-I and BRH-II, the crushing modes in the early and late stage of compression deformation are nearly the same. However, some differences can be seen from the dynamic response curve of the impact end. Considering that BRH-I has a larger wall thickness under the same mass, the stress level of BRH-I is higher than that of BRH-II when the "X" local shear zone appears in the early stage. With the continuous compression of the impact end, the cells of the whole honeycomb structure deform uniformly. It is easy to find that BRH-II has more cell walls than BRH-I (as shown in Fig. 2). The improvement of the strength of the whole structure is bound to improve the stress level. This is why the stress level of BRH-II may surpass BRH-I in the later stage of compression. However, all three honeycombs exhibit the same deformation mode under high-velocity impact as shown in Fig.8 (b). In the compression process, the local collapse first starts from the impact end, and then an "I"-shaped shear zone is gradually formed as the stress wave propagates to the distal end. Therefore, there is almost no difference in the plateau stress of the three honeycomb structures 
under high-velocity impact.

As another important indicator to measure crashworthiness, Fig. 9 shows the $S E A$ of these three honeycomb structures at different impact velocities. It can be found from Fig. 9 that under the impact velocity of $1 \mathrm{~m} / \mathrm{s}$ and $50 \mathrm{~m} / \mathrm{s}$, the $S E A$ of BRH-I and BRH-II have been greatly improved compared with the conventional RH. Specifically, the SEA of BRH-I has increased by $388.5 \%$ and $241.56 \%$. The growth rate of BRH-II even reaches $644.5 \%$ and $346.93 \%$. Under high-velocity impact, the inertia effect will play a leading role and the $S E A$ gap between these three honeycomb structures may be narrowed. BRH-I and BRH-II still have stronger energy absorption capacity with growth rates reaching about $42.08 \%$ and $51.49 \%$, respectively.

Besides, the negative Poisson's ratio effect caused by shrinkage deformation plays an important role in enhancing crashworthiness. As shown in Fig. 8, the shrinkage deformation feature of BRHI and BRH-II is different from the conventional RH. In order to better illustrate this difference, Fig. 10 shows the typical local deformation modes of single unit cell under quasi-static compression. It can be seen from Fig. 10 that the rotation of the plastic hinge causes the hypotenuse to bend and compact. The horizontal tensile force pulls the vertical plate, which further makes the structure shrink and deform. Compared with conventional RH, the difference is that BRH-I and BRH-II have a pair of plastic hinges with opposite rotation directions. This kind of plastic hinge in the opposite direction is more conducive to induce shrinkage deformation. Therefore, the shrinkage deformability of BRH-I and BRH-II is better than conventional RH. Meanwhile, the maximum horizontal strains of the three honeycombs under different impact velocities are extracted. For the calculation of NPR of each layer, six points are taken at the same interval on both sides of the structure. The average horizontal strain of this layer is calculated according to the horizontal displacement of the left and right points of each layer during the compression process. The NPR value of each layer is the ratio of the average horizontal strain to the vertical strain. The calculation formulas and its corresponding diagram are shown in Eqs. (13-15) and Fig. 11. As shown in Fig. 12, the maximum horizontal strains of BRH-I and BRH-II are larger than those of conventional RH under three different compression velocities. The maximum horizontal strains decrease with the increase of impact velocity. However, BRH-I and BRH-II are more sensitive to crushing velocity than conventional RH.

$$
\begin{gathered}
\varepsilon_{H}=\frac{x_{i} y_{j}-x_{i i} y_{j j}}{x_{i} y_{j}} \\
\varepsilon_{V}=\frac{\delta_{0}-\delta}{\delta_{0}} \\
v=\frac{\varepsilon_{H}}{\varepsilon_{V}}
\end{gathered}
$$

\section{Discussion}

According to the above discussion and analysis, it can be concluded that BRH has better mechanical properties and energy absorption capacity compared with conventional RH. BRH-I and BRH-II have similar compression characteristics and BRH-II has stronger energy absorption ability. Therefore, this section focuses on the study of the compression behavior and energy absorption characteristics of BRH-II. 


\subsection{Deformation mode}

\subsubsection{Deformation mode under different impact velocity}

It can be seen in Section. 4 that the energy absorption capacity of BRH is higher than that conventional RH due to the change of deformation mechanism. Considering that the deformation mode of honeycomb structure under different impact velocities may be different from each other, the crushing behavior of BRH-II under low, medium and high impact velocities is studied. The results show that there are three typical deformation modes for BRH-II, namely quasi-static, transition and dynamic collapse mode. A detailed analysis is made accordingly.

Fig. 13 shows the deformation mode of BRH-II under quasi-static compression. It can be seen from the map that during the compression process, plastic deformation propagates from the top or bottom to the center. The top and bottom ends have slight plastic deformation while the center position has more obvious plastic deformation. Severe plastic deformation appears in the central area of BRH-II with further compression, as shown in Fig. 13(d) and (e). Then, the overall plastic deformation occurs until it is finally compacted in Fig. 13(f). It is worth noting that the conventional $\mathrm{RH}$ always has various local shear bands in the process of dynamic compression. However, there are few local shear bands in the early stage of compression for BRH-II and the whole uniform plastic deformation occurs in the later stage until compaction. This is mainly due to the enhancement of the structural strength of unit cells and the plastic deformation of more cells will make the energy absorption capacity of BRH-II significantly improved.

Fig. 14 shows the deformation mode of BRH-II under $50 \mathrm{~m} / \mathrm{s}$ velocity impact. The influence of inertia effect is enhanced with the increase of impact velocity. The global plastic bending deformation in the quasi-static case $(V=1 \mathrm{~m} / \mathrm{s})$ disappears. It can be observed that BRH-II has a typical layer-by-layer failure process. At the beginning of compression, the upper half of the unit cells in the first row undergoes plastic deformation. After the upper half of the unit cells is fully compacted, the subsequent cells may deform in the same manner layer by layer. It's important to note that some triangular cells with smaller sides appear due to the introduction of the geometric characteristics of lotus leaf vein. There may be not enough space for the plastic buckling of cell walls in these triangular cells. The cell walls of these triangular cells do not collapse completely until densification and mix in the existed deformation band. In fact, this phenomenon also exists in the plastic deformation of BRH-II under quasi-static compression $(V=1 \mathrm{~m} / \mathrm{s})$, which can be found in Fig. 13.

The deformation mode of BRH-II under high-velocity impact is shown in Fig. 15. During the compression process, BRH-II first produced an I-shaped deformation mode at the impact end, and then this I-shaped deformation mode propagated vertically downward layer by layer. The characteristic of this compression mode is that only the single-layer unit cell undergoes dynamic compression until it is compacted, while the latter layer only undergoes slight plastic deformation, as shown in Fig. 15(c) and (d). It is worth noting that the unit cell of the latter layer will propagate downward with the I-shaped wave and transform from small plastic deformation to large plastic deformation. This is because the strength of the upper part of the BRH-II unit is much smaller than the lower part. As the compression displacement increases, the inertial effect will be gradually weakened.

\subsubsection{Deformation pattern diagram}

After the observation and analysis of the deformation modes of BRH-II under different impact velocities, it can be found that impact velocity may have a significant influence. Similar to most 
honeycomb structures, the deformation mechanism of BRH-II can be also summarized as quasistatic mode, transition mode and dynamic crushing mode. Fig. 16 shows the deformation modes of BRH-II with different relative densities under different impact velocities. It is noticeable that the deformation mode of BRH-II depends not only on the velocity, but also on the relative density. In the light of relative density $\bar{\rho}$, the corresponding empirical formula for the critical velocity can be obtained as follows:

$$
\begin{gathered}
V_{c 1}=10 \\
V_{c 2}=621.06 \bar{\rho}+13.98
\end{gathered}
$$

Where $V_{c 1}$ and $V_{c 2}$ represent the critical velocity of transition from quasi-static deformation pattern to transition mode and the critical velocity of transition from transition mode to dynamic mode, respectively.

\subsection{Plateau stress at impact end}

The stress-strain curve of in-plane compression of honeycomb structure is mainly divided into three stages, namely the initial linear elastic, the stable compression and the densification stage. Generally, the greater the plateau stress, the better the crashworthiness of the structure. Based on the corresponding stress-strain curve, all plateau stresses can be further calculated by Eq. (3). The stress is the ratio of the contact force between rigid plate and honeycomb structure to the contact area and the strain is the ratio of the compressed length of the structure to the original length. Fig. 17 shows the plateau stresses of BRH-II with different relative densities under different impact velocities. It can be seen that the increase of relative density and impact velocity will cause the increase of plateau stress. And there is a nonlinear curve relationship between the plateau stress and impact velocity. Therefore, according to the theoretical formula for calculating the static plateau stress in Ref. [34], it is assumed that the plateau stress of BRH-II under quasi-static compression can be calculated as follow:

$$
\sigma(\bar{\rho})=B \sigma_{y s} \bar{\rho}^{m}
$$

Considering that the impact energy can be almost completely converted into internal energy when impacted by $1 \mathrm{~m} / \mathrm{s}$, the kinetic energy can be ignored [34]. Therefore, the condition of $1 \mathrm{~m} / \mathrm{s}$ impact is considered as quasi-static condition. $B$ and $m$ in Eq. (11) are constants and can be obtained by least squares fitting. According to the data in Table. 1, the fitting parameters $B$ and $m$ are 0.5 and 2 , respectively. Fig. 18 shows the fitting results of the relative density of BRH-II and the corresponding plateau stress under quasi-static compression. The results show that Eq. (11) can well fit the plateau stress of BRH-II under quasi-static compression.

From the previous discussion of deformation modes under different impact velocities, it can be found that the influence of inertia effect increases with the increase of impact velocity. When the impact velocity reaches a certain value, the plastic deformation of BRH-II will propagate from top to bottom in a plane plastic wave-like deformation mode. Therefore, one-dimensional shock wave theory can be used to analyze the dynamic plateau stress of BRH-II. The theoretical model created by Reid [35,36] has been widely used in the analysis of various honeycomb structures, which can be expressed as follows:

$$
\sigma=\sigma_{0}+A V^{2}
$$

Where $\sigma, \sigma_{0}, V$ are dynamic plateau stress, static plateau stress and impact velocity, respectively. $A$ is a constant that can reflect the effect of inertia, and the expression is as follows:

$$
A=\frac{\bar{\rho} \rho_{S}}{\varepsilon_{d}}
$$


Where $\bar{\rho}, \rho_{s}, \varepsilon_{d}$ are the relative density, the density of the base material and the densification strain of BRH-II.

According to the data listed in Table. 1, the parameters in Eq. (12) are fitted by the least square method. Fig. 19 shows the plateau stress comparisons between numerical results and fitting results. The blue dot in the map is the numerical result and the red solid line is the fitting results. It is obvious that the simulated values are in good agreement with the fitting results. Table. 2 further lists the dynamic plateau stress mathematical models for BRH-II with different relative densities. It can be obtained from the table that the constant $A$ is $6.76 \times 10^{-5}, 1.01 \times 10^{-4}, 1.51 \times 10^{-4}, 2.25 \times 10^{-4}, 2.78 \times 10^{-4}$ and $3.29 \times 10^{-4}$ respectively, corresponding to different densities $(\bar{\rho}=0.044, \bar{\rho}=0.062, \bar{\rho}=0.089$, $\bar{\rho}=0.133, \bar{\rho}=0.177$ and $\bar{\rho}=0.222$. According to the least square method, the correlation relative density and constant $A$ of BRH-II can be further modeled as:

$$
A(\bar{\rho})=C_{1} \bar{\rho}^{2}+C_{2} \bar{\rho}+C_{3}
$$

Where $C_{1}, C_{2}$, and $C_{3}$ are fitting constants. Fig. 20 gives the relationship between the fitting results of constant $A$ and relative density. It can be found that Eq. (14) can accurately predict the value of constant $A$ under different relative densities. The fitting constants $C_{1}, C_{2}$, and $C_{3}$ obtained by the least squares method are respectively $-3.13 \times 10^{-3} 、 2.3 \times 10^{-3}$ and $-2.89 \times 10^{-5}$. Thus, the final expression of the dynamic plateau stress of BRH-II under different impact velocities can be expressed as follow:

$$
\sigma=0.5 \sigma_{y s} \bar{\rho}^{2}+\left(\left(-3.13 \times 10^{-3}\right) \bar{\rho}^{2}+\left(2.3 \times 10^{-3}\right) \bar{\rho}-2.89 \times 10^{-5} \quad\right) V^{2}
$$

\subsection{Shrinkage deformation and negative Poisson's ratio (NPR) effect}

In order to systematically study the shrinkage deformation characteristics of BRH-II, the horizontal strain of each layer of BRH-II under different impact velocities will be extracted for comparative analysis. The calculation formulas and its corresponding diagram are shown in Eqs. (13-15) and Fig. 11.

Fig. 21 shows the horizontal strain of each layer of BRH-II under low, medium and high impact velocities. It is noticeable that all the horizontal strains of each layer of BRH-II under different impact velocities show an upward trend with the increase of vertical strain. The first five layers of BRH-II have uneven horizontal strains at a fixed impact velocity, which are caused by uneven horizontal deformation. Quite the opposite, the sixth layer has almost no deformation. Under low and high velocity impact, the maximum horizontal strain appears in the third and fourth layers respectively. Meanwhile, the strain curves under medium and high-velocity impact are both smoother than those under low-velocity impact. Also note that under high-velocity impact, the shrinkage deformation ability of the first layer will not weakened like the other layers. This is due to the fact that effect of the plastic hinge with opposite rotation direction in the unit body (Fig. 10) is strengthened under high-velocity impact. Although the horizontal strain of BRH-II will be affected by the structural failure under high-velocity impact [16], because the strength of the upper half of the BRH-II unit is much smaller than the lower half, and this effect occurs before the lower half is densified, as shown in Fig. 14(c) and (d).

As shown in Fig. 21(a), the horizontal strains of the third or fourth layers of BRH-II in the early stage of low-velocity impact are all greater than other layers. This is due to the appearance of the $\mathrm{X}$-shaped deformation mode, which results in a large shrinkage deformation of the middle layer. Under medium and high velocities, the horizontal strain of the $(\mathrm{N}-1)^{\text {th }}$ layer of BRH-II at the initial stage of impact is greater than that of the $\mathrm{N}^{\text {th }}$ layer, which is consistent with the layer-by-layer 
deformation mechanism.

\subsection{Energy absorption capacity}

As stated above, $S E A$ is also an important index for evaluating crashworthiness. Therefore, in this section the energy absorption capacity of BRH-II is further studied. Fig. 22 shows the influence of the impact velocity and relative density on $S E A$ of BRH-II. In order to show that the impact velocity and relative density of BRH-II have a coupling effect on the $S E A$, contour map of the SEA under different impact velocities and relative densities is given in Fig. 22(b). As shown in Fig 22(a), the $S E A$ of BRH-II keeps increasing as the impact velocity and relative density increases. However, as the impact velocity increases, the SEA of BRH-II under different relative densities tends to gradually converge. It demonstrates that the $S E A$ of BRH-II is more sensitive to impact velocity at low relative density, and the influence of relative density on the energy absorption capacity of BRHII will gradually weaken with the increase of impact velocity.

Considering that the structural parameters have a very important influence on its crashworthiness, Fig. 23 shows the plateau stress and SEA of BRH-II with different branch angles $\beta$, and the unit cells of BRH-II under different branch angles $\beta$ correspondingly are shown. As shown in Fig. 23, the plateau stress shows an upward trend with the increase of $\beta$, while $S E A$ first increases and then decreases. This is mainly because that when the branch angle is greater than $74^{\circ}$, although the total energy absorption can be increased with the further increase of $\beta$, the increments in total energy absorption is smaller than the mass increments.

Meanwhile, considering that the BRH-II unit is a up-down asymmetric structure, it is very meaningful to study its arrangement. In order to study the impact of BRH-II arrangement on its crashworthiness, five different arrangements (a) (b) (c) (d) (e) are designed, as shown in Fig. 24. It can be seen from Fig. 25 that different arrangements of BRH-II have different $S E A$ and plateau stresses, and arrangement (e) has the largest $S E A$ and plateau stress values. It shows that different arrangements have a very important influence on the $S E A$ and plateau stress of BRH-II.

\section{Conclusions}

Inspired by the biological structure of nature, this paper chooses the lotus leaf vein as the biological prototype and designs a novel bio-inspired re-entrant honeycomb $(\mathrm{BRH})$ with negative Poisson's ratio. The compression behavior and energy absorption capacity of BRH-I, BRH-II and RH under different impact velocities are compared based on finite element numerical simulation. Subsequently, the mechanical properties of BRH-II are systematically studied. The following conclusions can be drawn:

(1) BRH has greater plateau stress and energy absorption capacity than conventional RH, especially at impact velocities of $1 \mathrm{~m} / \mathrm{s}$ and $50 \mathrm{~m} / \mathrm{s}$. The $S E A$ of BRH-I is respectively $388.5 \%$ and $241.56 \%$ higher than conventional RH, and the growth rate of BRH-II respectively reaches $644.5 \%$ and $346.93 \%$. Under the $100 \mathrm{~m} / \mathrm{s}$ high-velocity impact, the $S E A$ gap between the three honeycomb structures has narrowed, but BRH-I and BRH-II still have outstanding energy absorption capacity with growth rates reaching $42.08 \%$ and $51.49 \%$, respectively. In addition, BRH shows better shrinkage deformation characteristics than conventional RH.

(2) The deformation modes of BRH-II can be divided into three types, namely quasi-static mode, transition mode and dynamic crushing mode. Under low and medium velocity impact, the honeycomb structure shows overall plastic deformation. The obvious $\mathrm{X}$-shaped local shear zone 
first appears in the quasi-static crushing mode. When the impact velocity is high enough, inertia effect plays a leading role and the "I"-shaped local deformation band can be observed at the impact end. The empirical formula of critical velocity is then given based on the classification diagram of BRH-II.

(3) The plateau stress and SEA of BRH-II increase with the increase of relative density and impact velocity. The $S E A$ of BRH-II is more sensitive to impact velocity at low relative density, and the influence of relative density will gradually weaken with the increase of impact velocity. The empirical formulas are given to predict the dynamic plateau stress of BRH-II at the impact end. In addition, the branch angle $\beta$ and arrangement of BRH-II have an important influence on its plateau stress and energy absorption capacity.

(4) BRH-II shows different shrinkage deformation characteristics and negative Poisson's ratio effects under different impact velocities. Under the same impact velocity, there is a big difference in the horizontal strain between the layers of BRH-II. At higher impact velocities, the shrinkage deformation of the $(\mathrm{N}-1)^{\text {th }}$ layer is earlier than that of the $\mathrm{N}^{\text {th }}$ layer.

\section{Availability of data and materials}

Not applicable

\section{Competing interests}

The authors declare no competing financial interests.

\section{Funding}

Supported by National Natural Science Foundation of China (Grant No.51705215, NO. 52005230). The China Postdoctoral Science Foundation funded project (NO. 2020M681531).

\section{Authors' contributions}

Yonghui Wang and Qiang He conceived and designed the study; Yonghui Wang and Hang Gu performed the simulations and wrote the initial manuscript; Yu Chen assisted with the results analysis; Qiang He and Honggen Zhou reviewed and edited the manuscript. All authors read and approved the final manuscript.

\section{Authors' Information}

Yonghui Wang, born in 1994, is currently a master candidate at the School of Mechanical Engineering, Jiangsu University of Science and Technology, China. His research interest is mechanical properties of thin-walled structures.

Qiang He, born in 1989, is currently an associate professor at the School of Mechanical Engineering, Jiangsu University of Science and Technology, China. He received his $\mathrm{PhD}$ degree from Nanjing University of Science and Technology, China, in 2016. Her research interest is mechanical behavior analysis of lightweight materials and structures.

Yu Chen, born in 1987, is currently a lecturer at the School of Mechanical Engineering, Jiangsu Institute of Technology, China. He received his PhD degree from Nanjing University of Science and Technology, China, in 2017. Her research interest is dynamic analysis of complex mechanism Hang Gu, born in 1995, is currently a master candidate at the School of Mechanical Engineering, Jiangsu University of Science and Technology, China. His research interest is mechanical properties 
of thin-walled structures.

Honggen Zhou, born in 1976, is currently a full professor at the School of Mechanical Engineering, Tongji University, China. His main research interest is structural dynamic analysis.

\section{Author Details}

1 School of Mechanical Engineering, Jiangsu University of Science and Technology, Zhenjiang, 212000, China

2 School of Mechanical Engineering, Jiangsu Institute of Technology, Changzhou, 213001, China

\section{Acknowledgement}

This work was financially supported by The National Natural Science Foundation of China (No. 51705215, NO.52005230), The China Postdoctoral Science Foundation funded project (NO. 2020M681531). The authors would like to express their thanks.

\section{Reference}

[1] DB Xiao, XQ Chen, Y Li, WW Wu, Fang DN, The structure response of sandwich beams with metallic auxetic honeycomb cores under localized impulsive loading-experiments and finite element analysis, Mater. Des. 176 (2019) 107840.

[2] Y Wu, J Fang, Y He, W Li, Crashworthiness of hierarchical circular-joint quadrangular honeycombs, Thin-Walled Struct. 133 (2018) 180-191.

[3] X Yang, J Ma, Y Shi, Y Sun, J Yang, Crashworthiness investigation of the bio- inspired bidirectionally corrugated core sandwich panel under quasi-static crushing load, Mater. Des. 135 (2017) 275-290.

[4] L Dong, V Deshpande, H Wadley. Mechanical response of Ti-6Al-4V octet-truss lattice structures. Int J Solids Struct 2015;60-61:107-24.

[5] H Liu, Z Zhang, H Liu, J Yang. Effect of elastic target on Taylor-Hopkinson impact of lowdensity foam material. Int J Impact Eng 2016;94:109-19.

[6] LL Hu, TX Yu, ZY Gao, XQ Huang. The inhomogeneous deformation of polycarbonate circular honeycombs under in-plane compression. Int J Mech Sci 2008;50(7):1224-36.

[7] G Cricrì, M Perrella, C Calì. Honeycomb failure processes under in-plane loading. Compos B 2013;45:1079-90.

[8] XF Yang, JX Ma, YX Sun, JL Yang. Ripplecomb: a novel triangular tube reinforced corrugated honeycomb for energy absorption. Compos Struct 2018;202: 988-99.

[9] S Liang, HL Chen. Investigation on the square cell honeycomb structures under axial loading. Compos Struct 2006;72: 446-54.

[10] J Ju, JD Summers, Compliant hexagonal periodic lattice structures having both high shear strength and high shear strain, Mater. Des. 32 (2) (2011) 512-524.

[11] HL Tan, ZC He, KX Li, E Li, AG Cheng, B Xu, In-plane crashworthiness of re- entrant hierarchical honeycombs with negative Poisson's ratio, Compos. Struct. 229 (2019) 111415.

[12] C Qi, A Remennikov, LZ Pei, S Yang, ZH Yu, TD Ngo, Impact and close-in blast response of auxetic honeycomb-cored sandwich panels: experimental tests and numerical simulations, Compos. Struct. 180 (15) (2017) 161-178.

[13] RS Lakes, Foam structures with a negative Poisson's ratio, Science 235 (1987) 1038-1040. 
[14] X Hou, H Hu, V Silberschmidt, Numerical analysis of composite structure with in-plane isotropic negative Poisson's ratio: effects of materials properties and geometry features of inclusions, Composer Part B 58 (2014) 152-159.

[15] H Wang, Y Zhang, W Lin, Q Qin, A novel two-dimensional mechanical metamaterial with negative Poisson's ratio, Comput. Mater. Sci. 171 (2020) 109232.

[16] Q Gao, L Wang, Z Zhou, Z Ma, C Wang, Y Wang, Theoretical, numerical and experimental analysis of three-dimensional double-V honeycomb, Mater. Des. 139 (2018) 380-391.

[17] H Qin, D Yang, C Ren, Modelling theory of functional element design for metamaterials with arbitrary negative Poisson's ratio, Comput. Mater. Sci. 150 (2018) 121-133.

[18] D Xiao, X Kang, Y Li, W Wu, J Lu, G Zhao, Fang D, Insight into the negative Poisson's ratio effect of metallic auxetic reentrant honeycomb under dynamic compression, Mater. Sci. Eng. A 763 (2019) 138151.

[19] LL Hu, MZ Zhou, H Deng, Dynamic crushing response of auxetic honeycombs under large deformation: theoretical analysis and numerical simulation, Thin-Walled Struct. 131 (2018) 373384.

[20] HL Tan, ZC He, KX Li, Eric Li, AG Cheng, Bing Xu, In-plane crashworthiness of re-entrant hierarchical honeycombs with negative Poisson's ratio, Composite Structures. 229 (2019) 111415.

[21] XF Yang, XL Xi, QF Pan, H Liu, In-plane dynamic crushing of a novel circular-celled honeycomb nested with petal-shaped mesostructure, Composite Structures. 226 (2019) 111219.

[22] HF Yin, YY Xiao, GL Wen, et al. Crushing analysis and multi-objective optimization design for bionic thin-walled structure. Materials and Design, 87 (2015) 825-834.

[23] Ngoc San Ha, Lu GX. A review of recent research on bio-inspired structures and materials for energy absorption applications. Compos Part B 2020(181): 107496.

[24] M Zou, SC Xu, CG Wei, et al. A bionic method for the crashworthiness design of thin-walled structures inspired by bamboo. Thin-Walled Structures, 2016(101):222-230.

[25] JF Song, SC Xu, HX Wang, et al. Bionic design and multi-objective optimization for variable wall thickness tube inspired bamboo structures. Thin-Walled Structures, 2018(125): 76-88.

[26] HY Jiang, YR Ren, et al. Crashworthiness of novel concentric auxetic reentrant honeycomb with negative Poisson's ratio biologically inspired by coconut palm. Thin-walled Structures, 2020. 154 (2020) 106911.

[27] S Liu, Z Tong, Z Tang, Y Liu, Z Zhang, Bionic design modification of non-convex multi-corner thin-walled columns for improving energy absorption through adding bulkheads, Thin-Walled Struct. 88 (2015) 70-81.

[28] SHA Sabah, ABH Kueh, MY Al-Fasih, Comparative low-velocity impact behavior of bioinspired and conventional sandwich composite beams, Compos. Sci. Technol. 149 (2017) 64-74.

[29] J Xiang, J Du, D Li, F Scarpa, Numerical analysis of the impact resistance in aluminum alloy bi-tubular thin-walled structures designs inspired by beetle elytra, J. Mater. Sci. 52 (22) (2017) 13247-13260.

[30] JS Shang, NHH Ngern, VBC. Tan, Crustacean-inspired helicoidal laminates, Compos. Sci. Technol. 128 (2016) 222-232.

[31] Q Liu, J Ma, Z He, Z Hu, D Hui, Energy absorption of bio-inspired multi-cell CFRP and aluminum square tubes, Composer Part B 121 (2017) 134-144.

[32] H Jiang, Y Ren, Z Liu, S Zhang, Z Lin. Low-velocity impact resistance behaviors of bio- 
inspired helicoidal composite laminates with non-linear rotation angle based layups, Compos. Struct. 214 (2019) 463-475.

[33] LL Hu, MZ Zhou, H Deng, Dynamic crushing response of auxetic honeycombs under large deformation: theoretical analysis and numerical simulation, Thin-Walled Struct. 131 (2018) 373384.

[34] DH Zhang, QG Feic, PW Zhang, In-plane dynamic crushing behavior and energy absorption of honeycombs with a novel type of multi-cells, Thin-Walled Structures. 117 (2017) 199-210.

[35] Zou Z, Reid SR, Tan PJ, Li S, Harrigan JJ. Dynamic crushing of honeycombs and features of shock fronts. Int J Impact Eng. 2009; 36:165-76.

[36] SR Reid, Peng C, Dynamic uniaxial crushing of wood. International Journal of Impact Engineering,1997, 19: 531-570. 
Figures

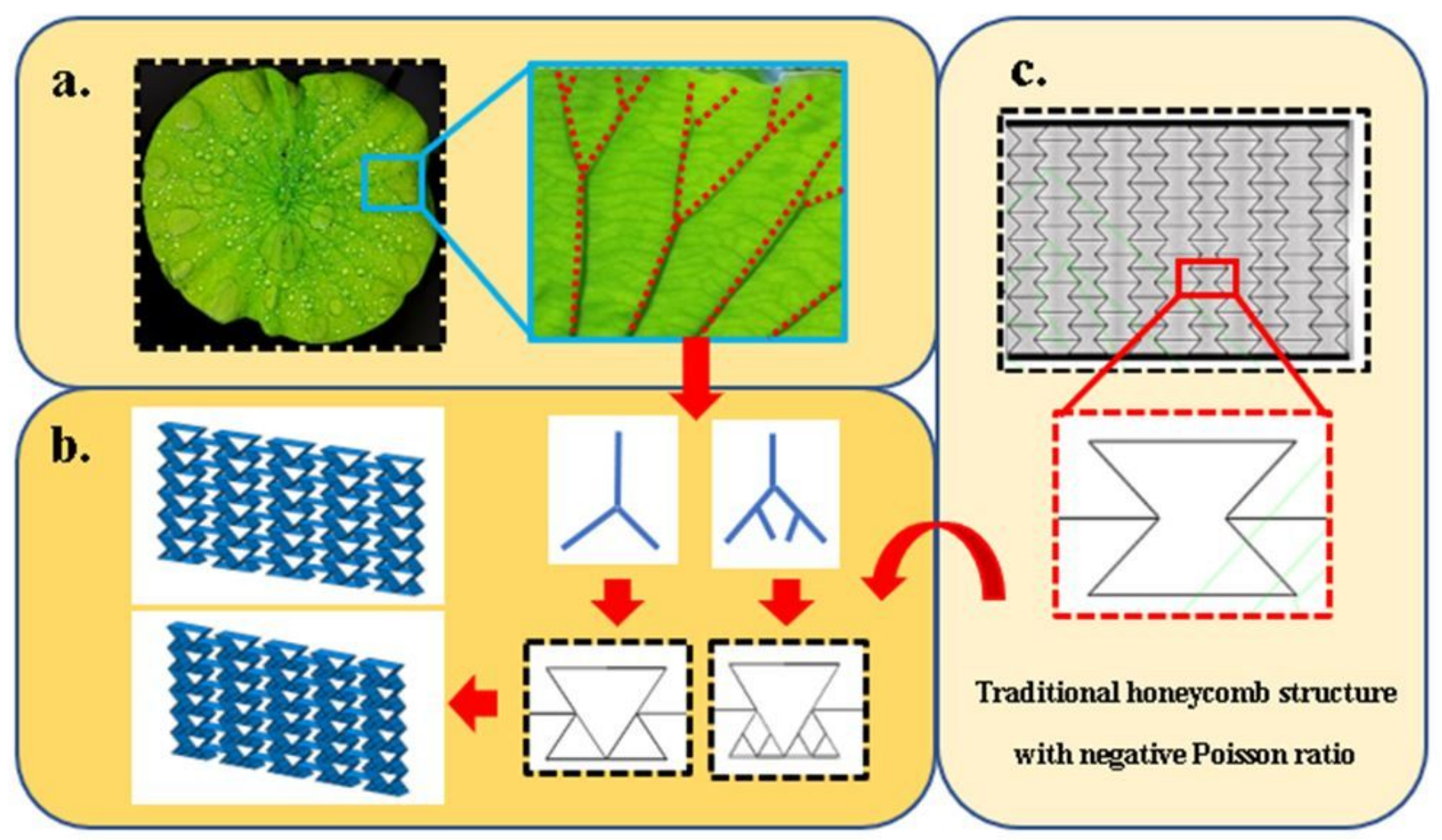

Figure 1

Bio-inspired re-entrant honeycomb $(\mathrm{BRH})$ inspired by leaf vein branched structure.

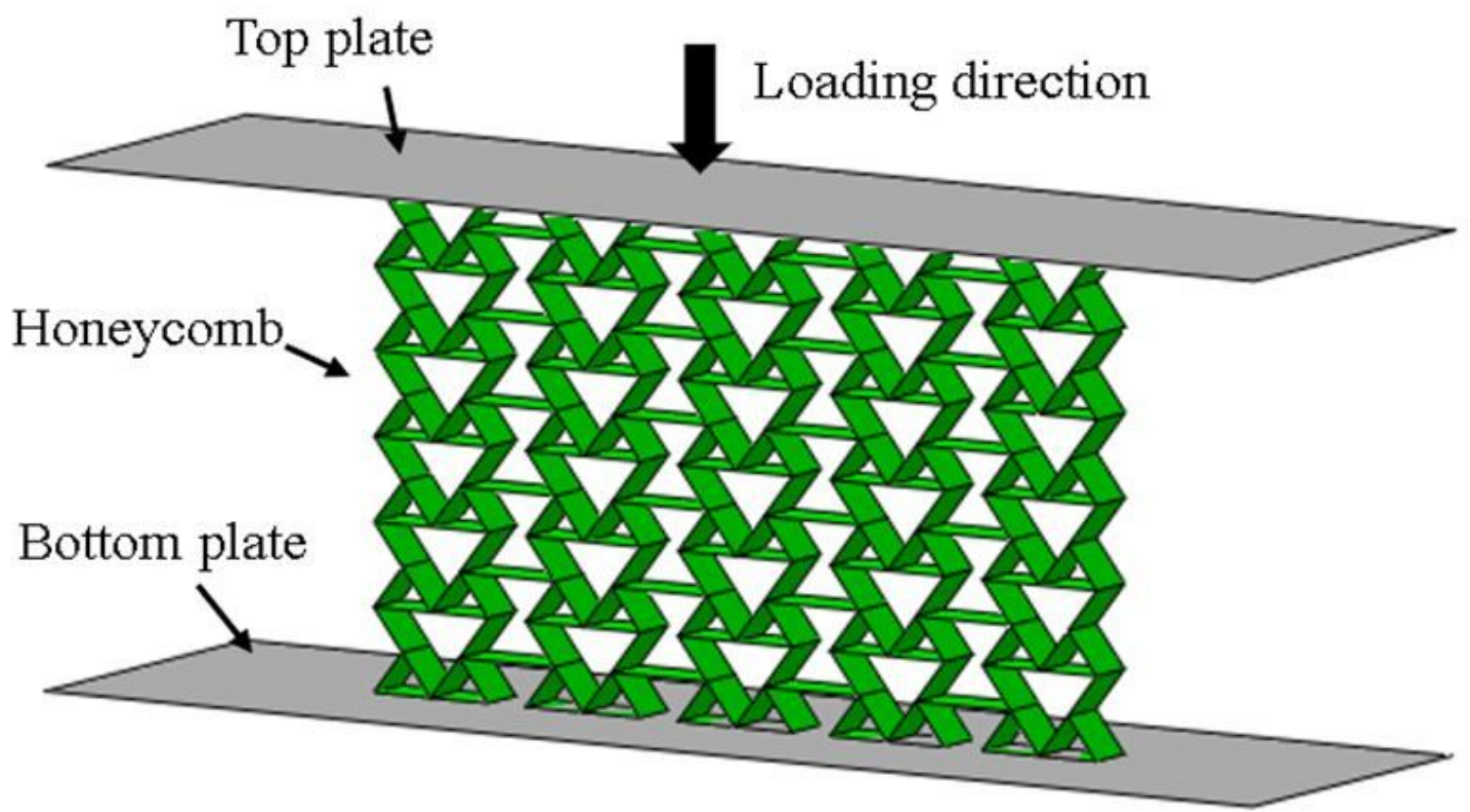




\section{Figure 3}

Finite element model of BRH subjected to in-plane crushing.

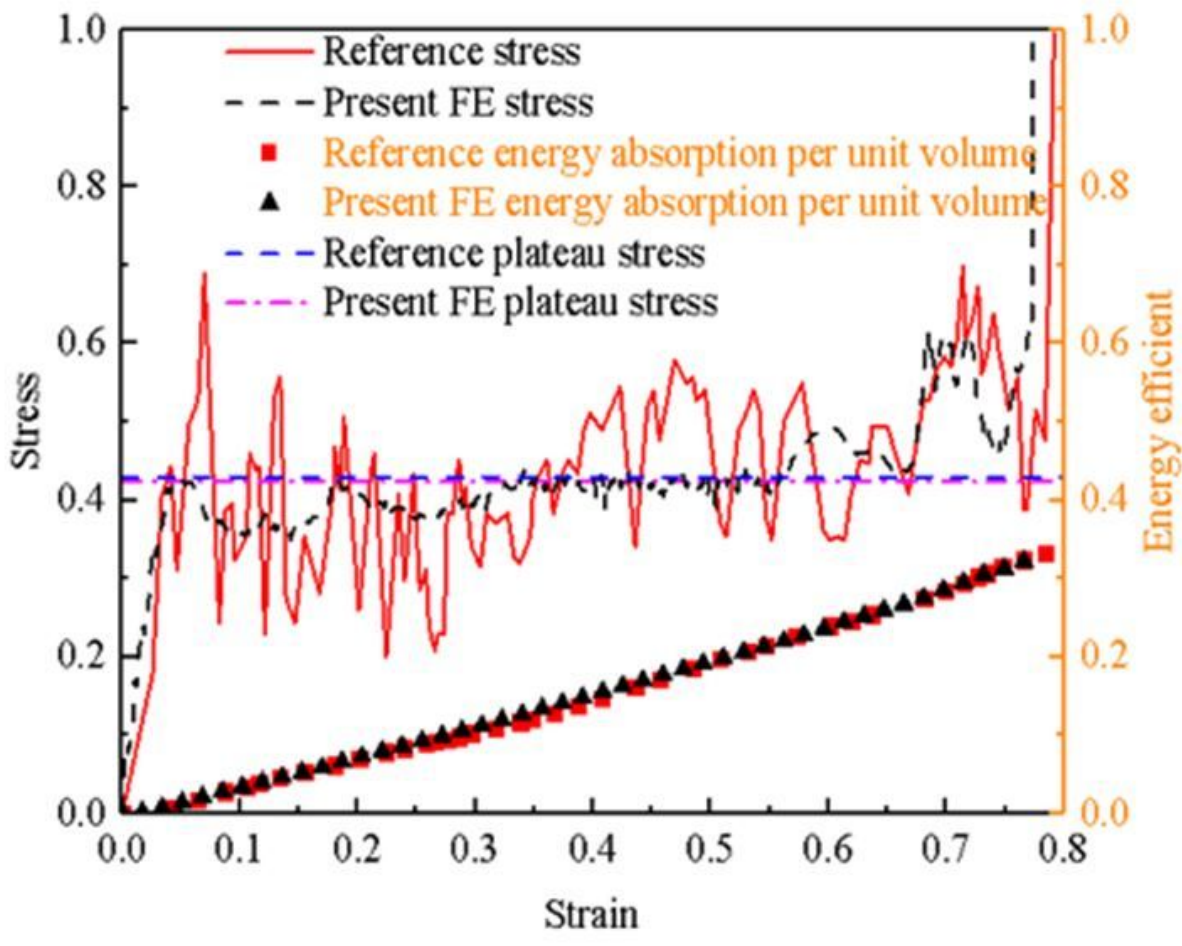

(a)

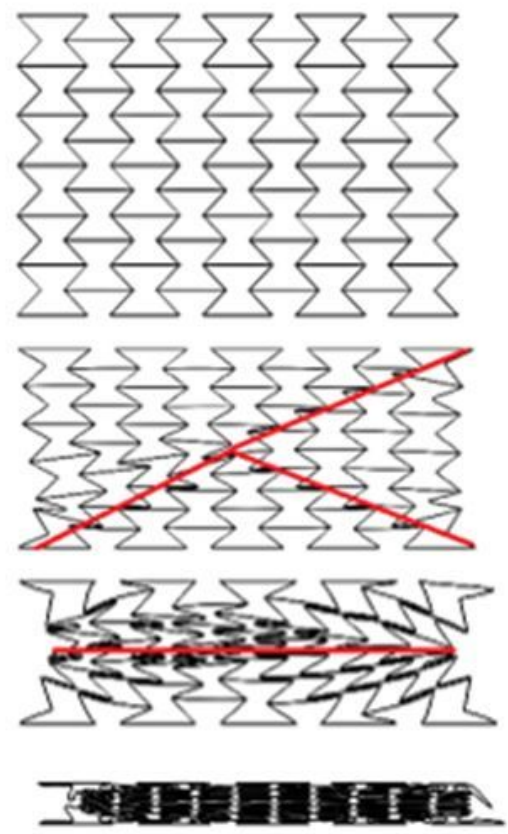

(b)

\section{Figure 4}

Comparison between the simulation results and Ref. [16] for RH under quasi-static compression: (a) dynamic response curve and energy absorption; (b) deformation mode

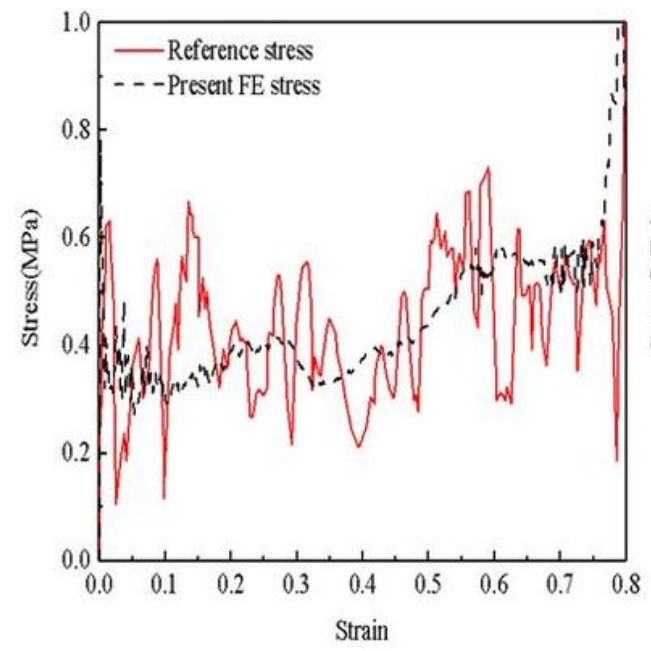

(a)

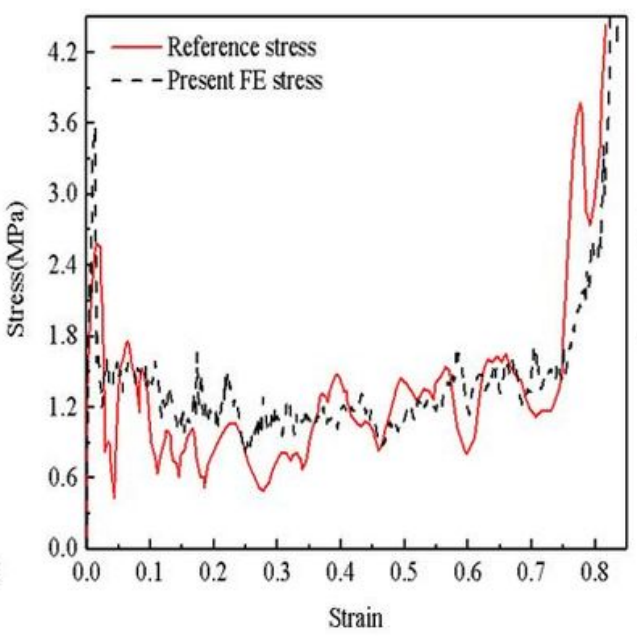

(b)

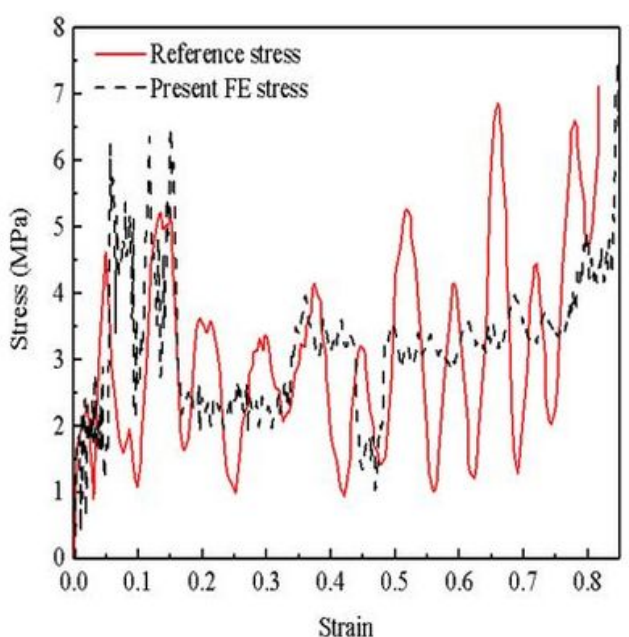

(c)

Figure 5 
Comparison of dynamic response curves under different impact velocities: (a) Low velocity $(10 \mathrm{~m} / \mathrm{s})$; (b) Medium velocity $(50 \mathrm{~m} / \mathrm{s})$; (c) High velocity $(100 \mathrm{~m} / \mathrm{s})$.

\begin{tabular}{|c|c|c|c|}
\hline \multirow{2}{*}{ Strain } & Low velocity $(10 \mathrm{~m} / \mathrm{s})$ & Medium velocity $(50 \mathrm{~m} / \mathrm{s})$ & High velocity $(100 \mathrm{~m} / \mathrm{s})$ \\
\hline & FE $\quad$ Reference[16] & FE Reference[16] & FE Reference[16] \\
\hline 0.2 & 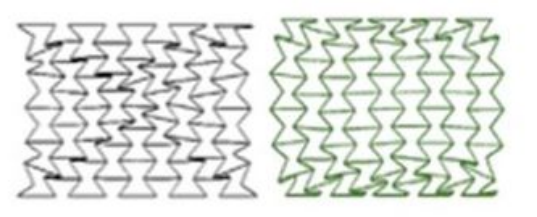 & 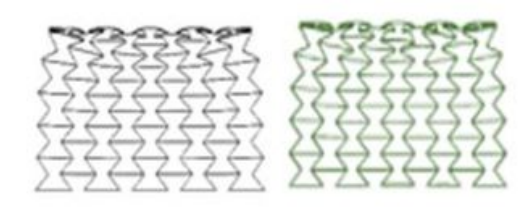 & 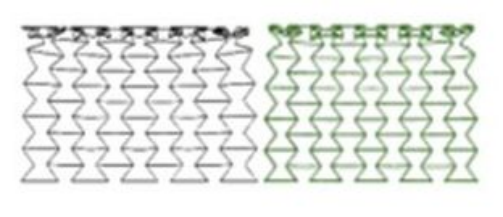 \\
\hline 0.4 & 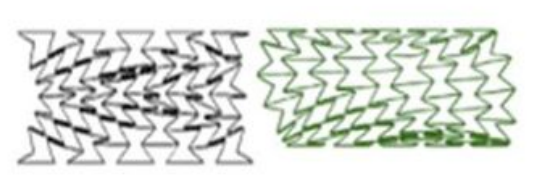 & 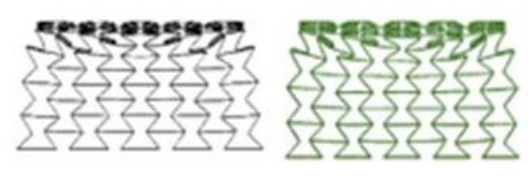 & 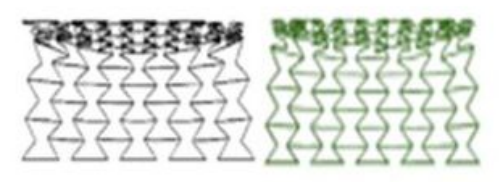 \\
\hline 0.6 & 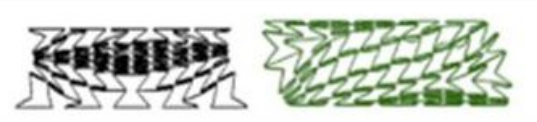 & 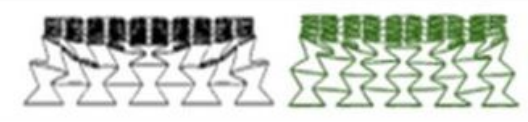 & 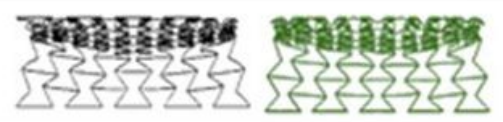 \\
\hline 0.8 & 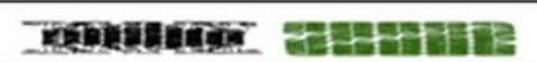 & 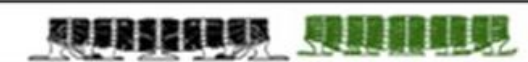 & 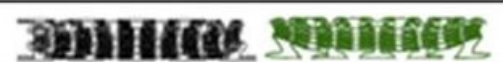 \\
\hline
\end{tabular}

\section{Figure 6}

Comparison of deformation modes under different impact velocities 


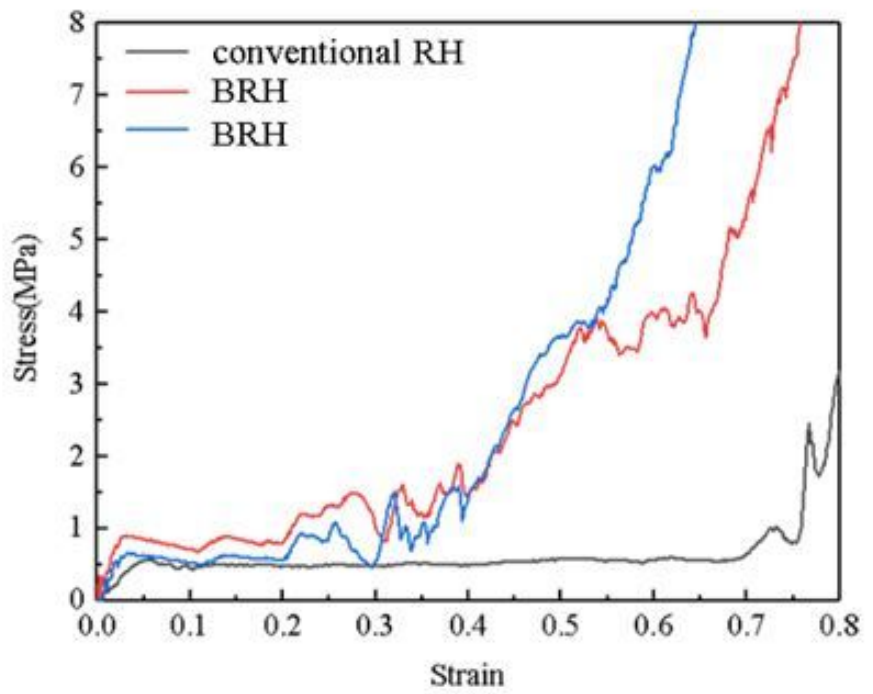

(a) $V=1 \mathrm{~m} / \mathrm{s}$

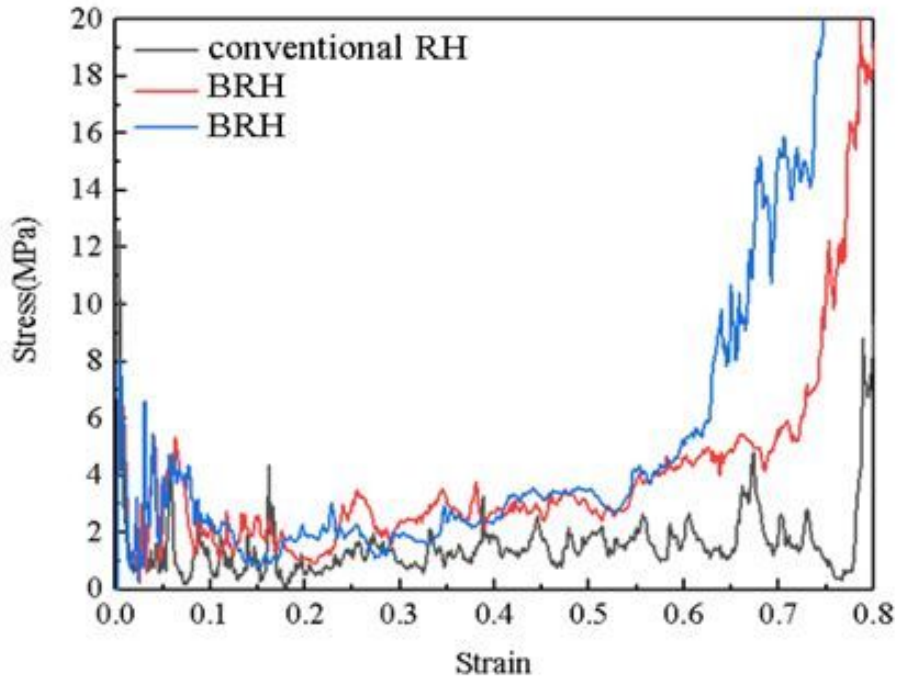

(b) $V=50 \mathrm{~m} / \mathrm{s}$

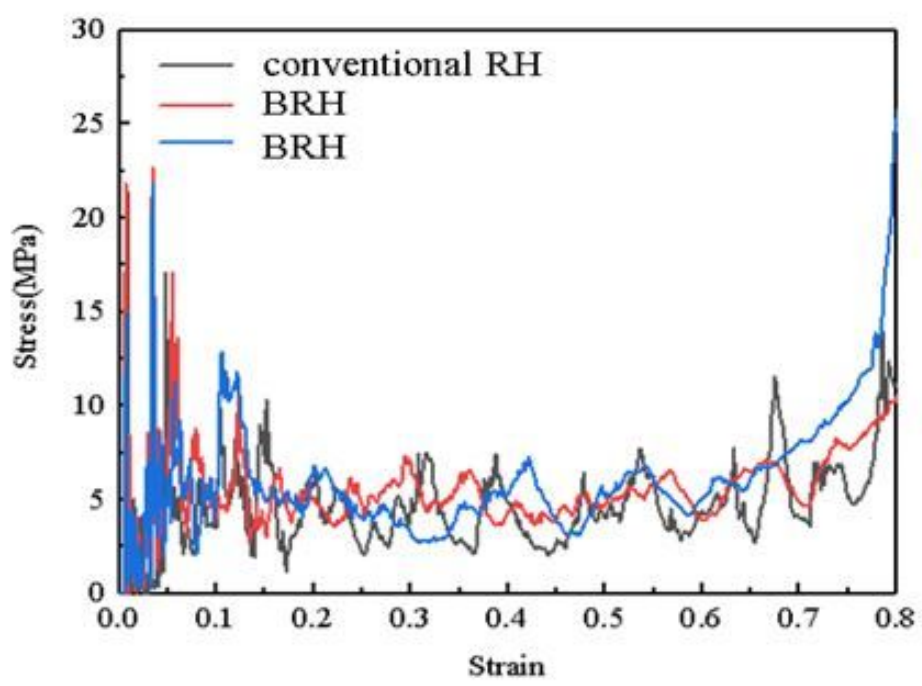

(c) $V=100 \mathrm{~m} / \mathrm{s}$

\section{Figure 7}

Stress-strain curves of three honeycomb structures under different impact velocities. 

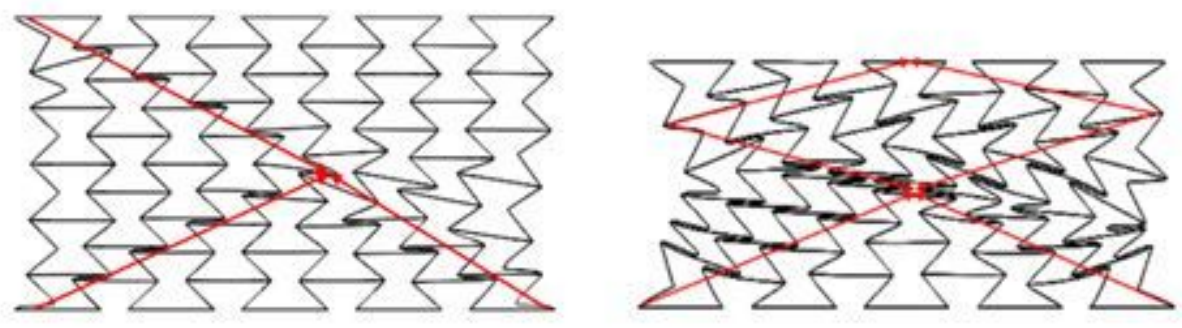

\section{Conventional RH}
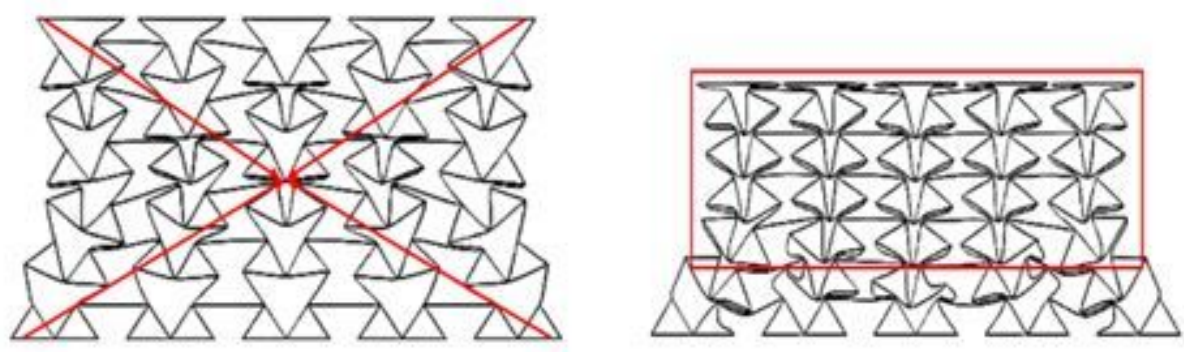

BRH-I

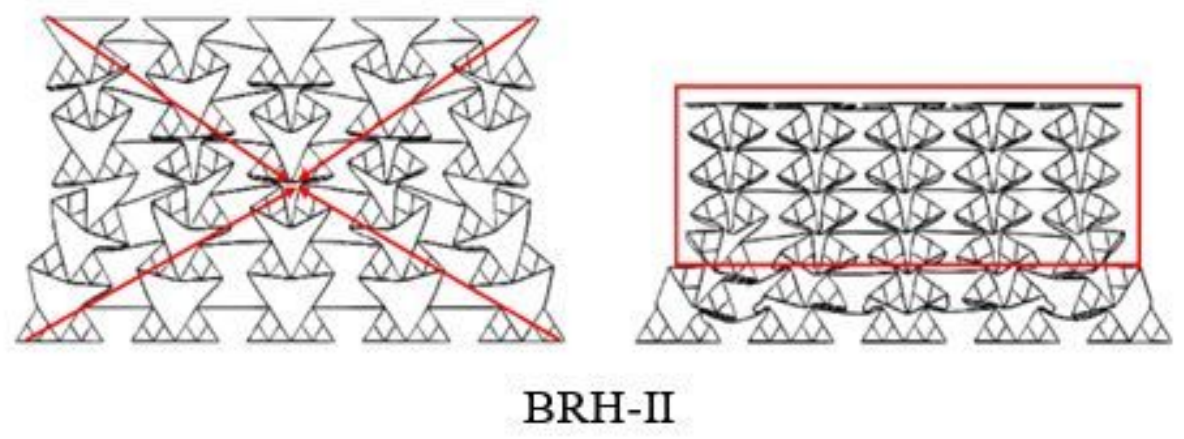

(a) Deformation modes of honeycombs under low-velocity impact
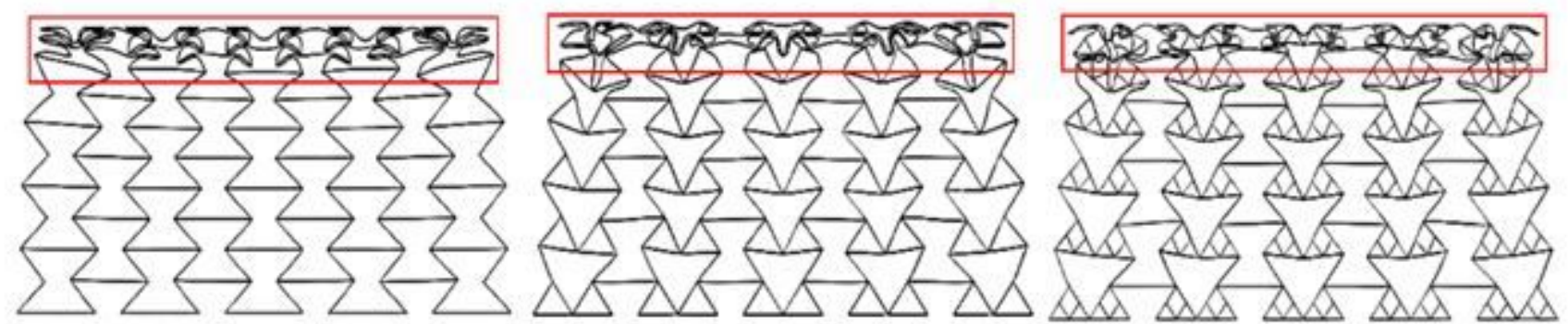

(b) Deformation modes of honeycombs under high-velocity impact

\section{Figure 8}

Deformation modes of honeycombs under different impact velocities. 


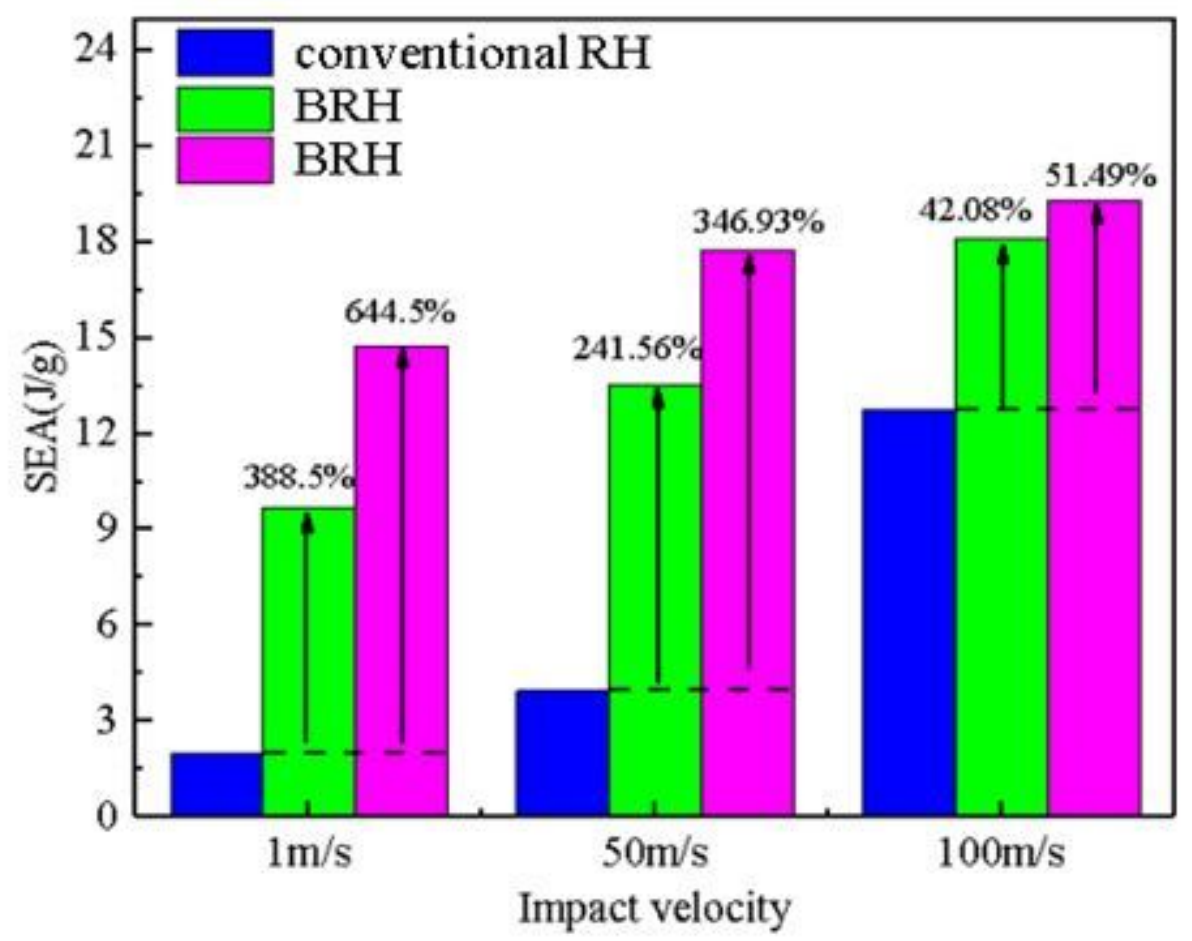

Figure 9

SEA of honeycomb structures under different impact velocities

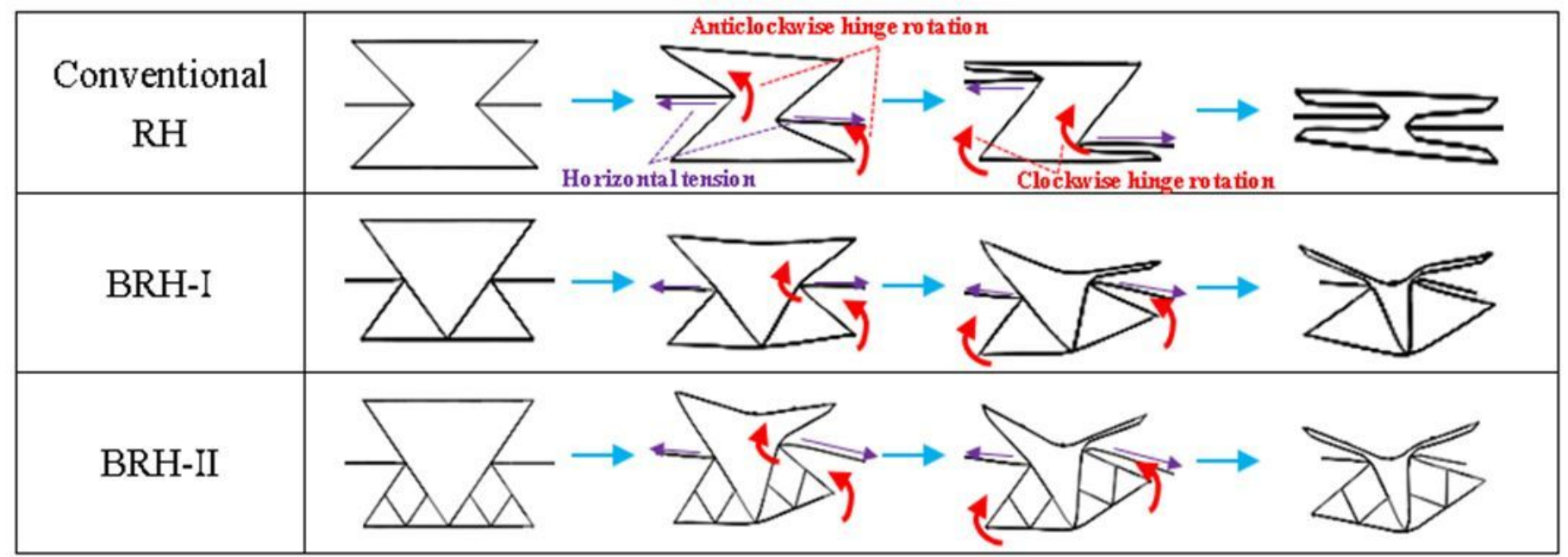

Figure 10

Typical local deformation mode of single unit cell under quasi-static compression 

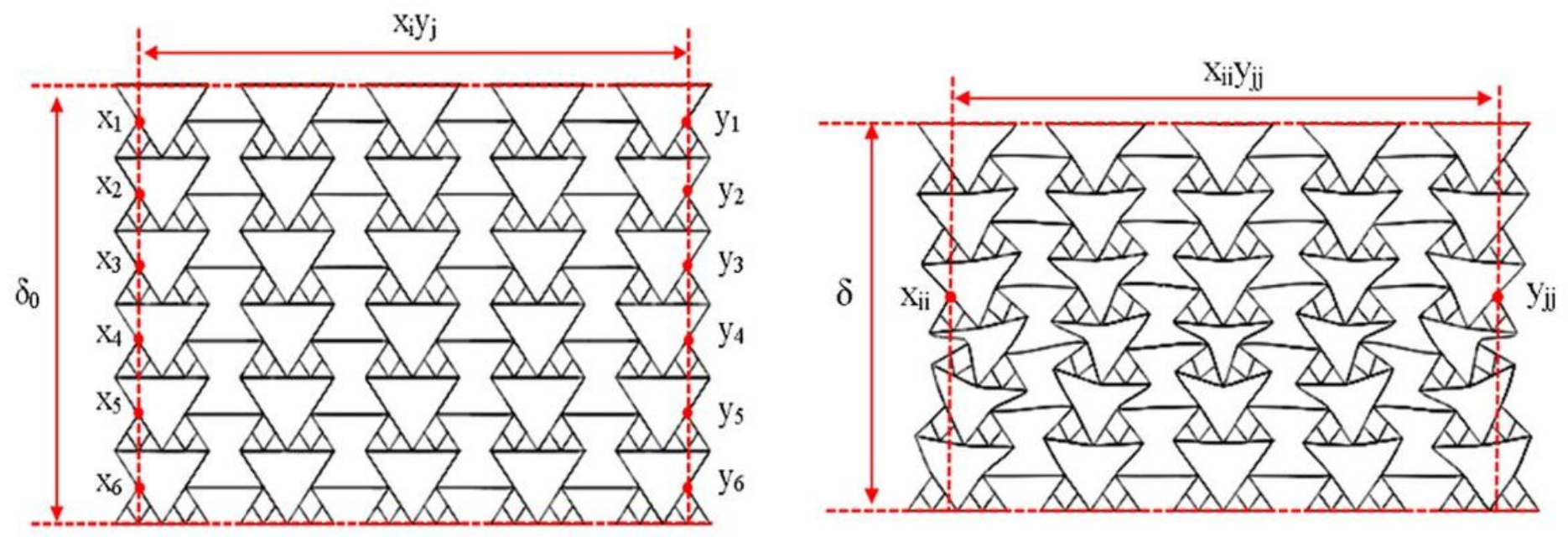

Figure 11

The calculation of $\mathrm{BRH}-\mathbb{Q}$ horizontal strain and vertical strain.
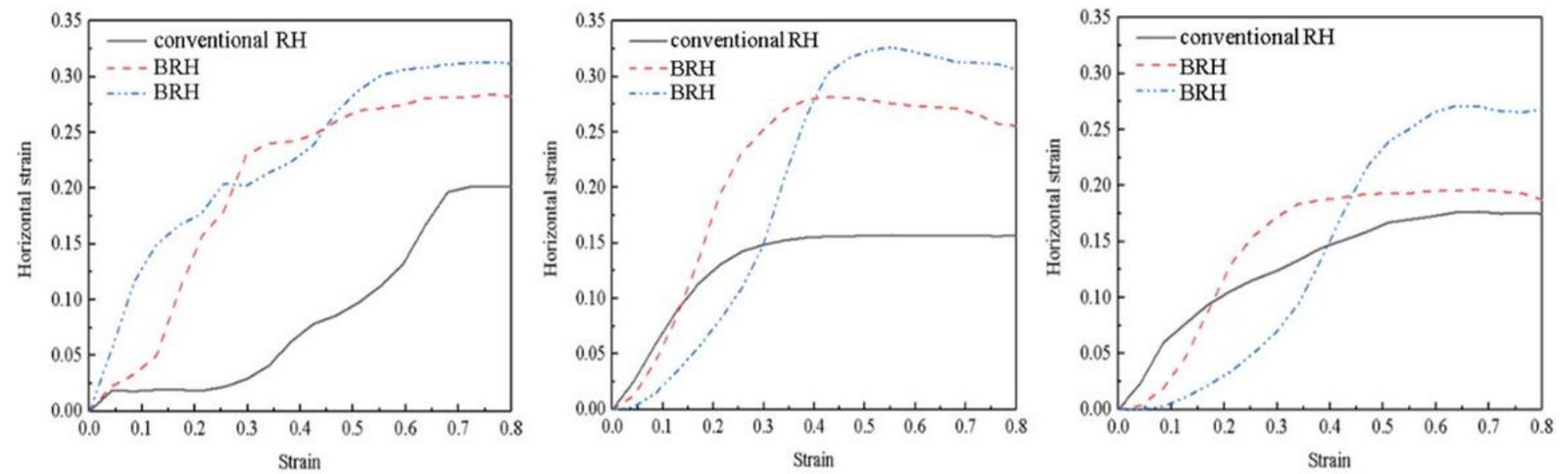

Figure 12

Maximum horizontal strain of honeycomb structure under different impact velocities. 


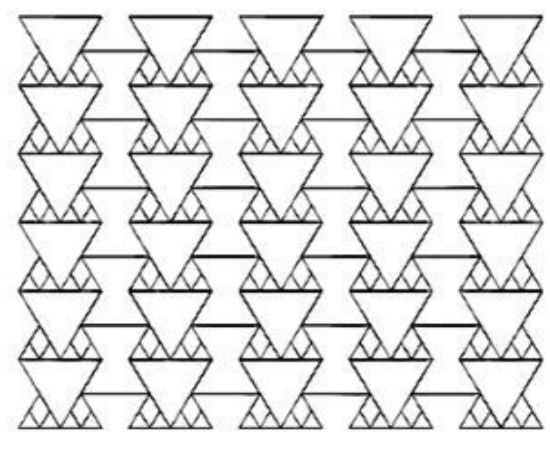

(a) $\varepsilon=0$

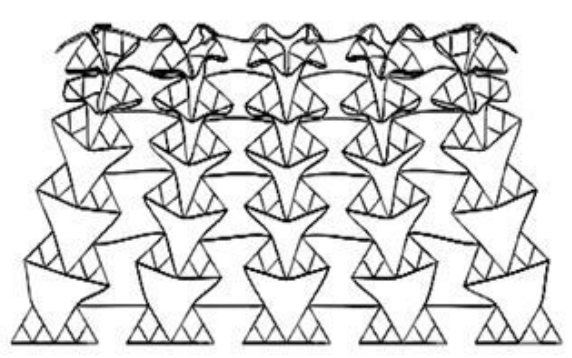

(d) $\varepsilon=0.3$

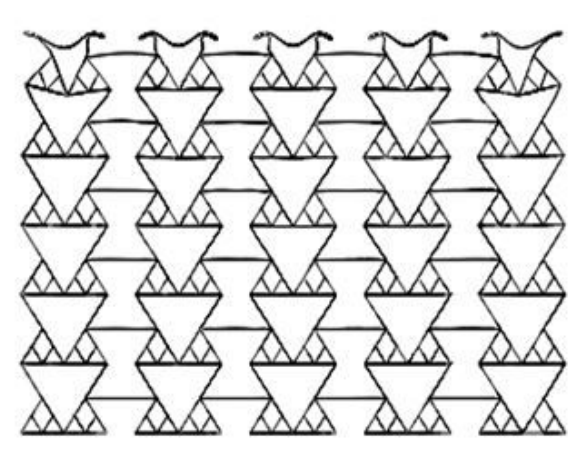

(b) $\varepsilon=0.043$

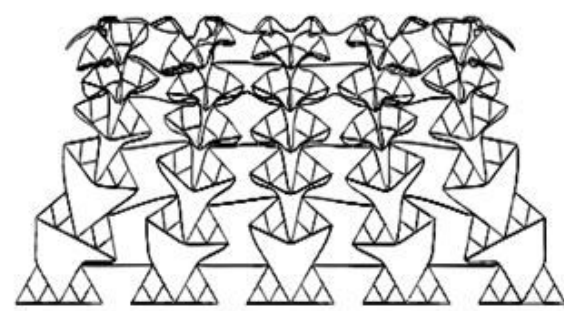

(e) $\varepsilon=0.382$

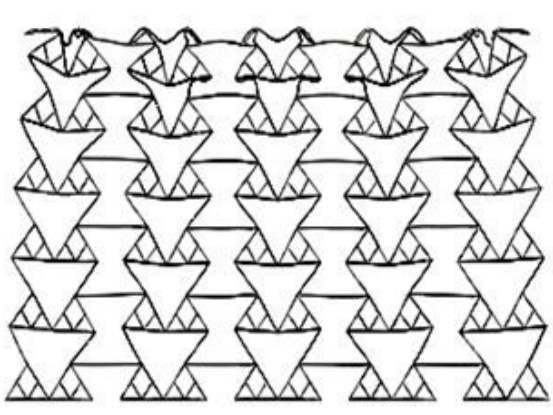

(c) $\varepsilon=0.127$

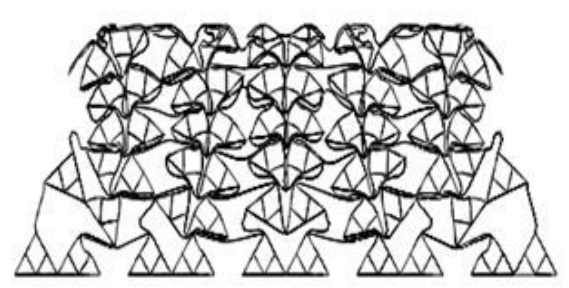

(f) $\varepsilon=0.51$

Figure 14

please see the manuscript file for the full caption 


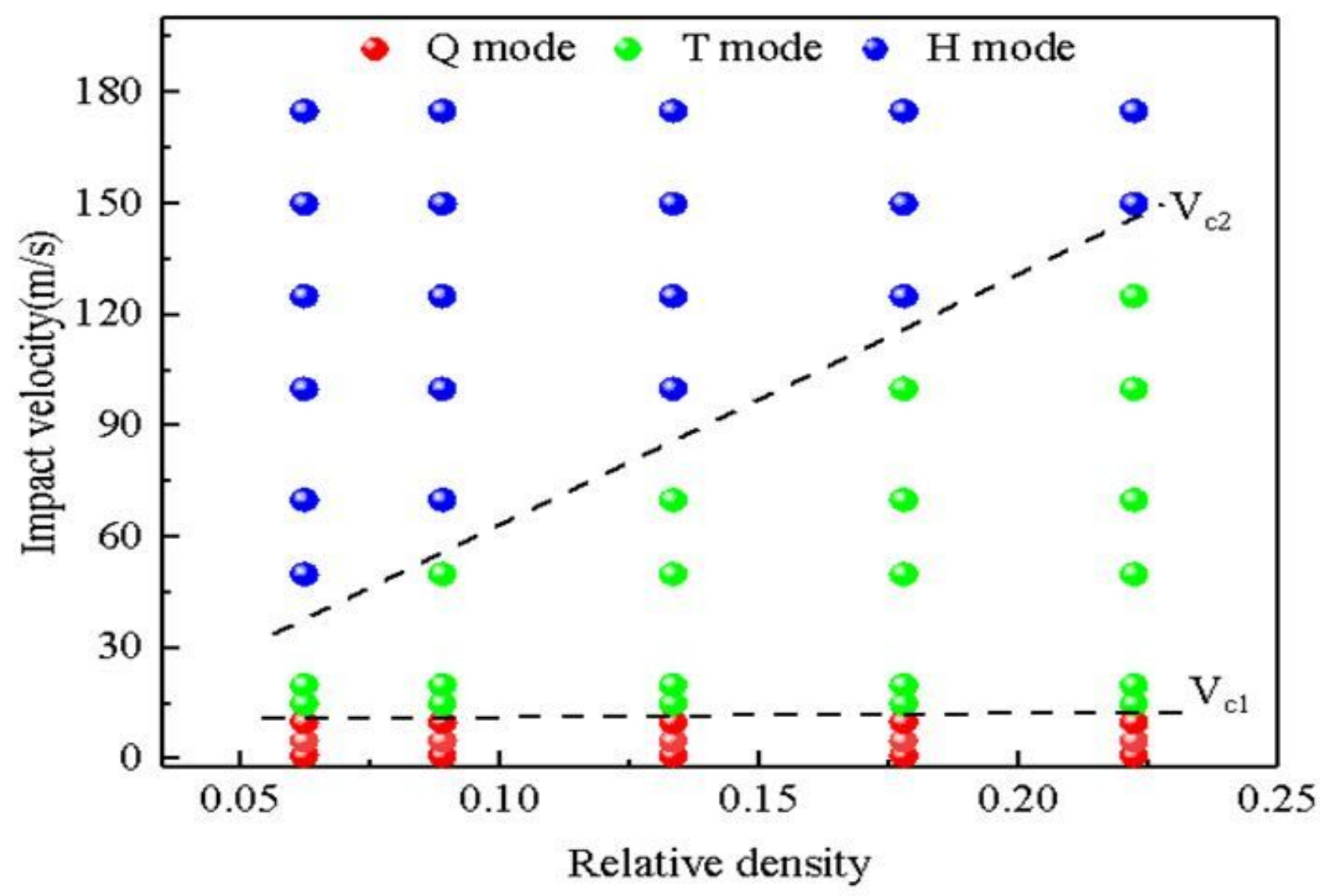

Figure 16

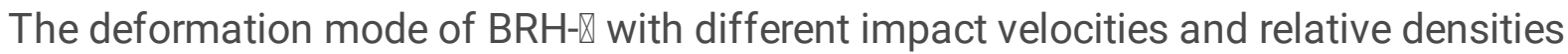




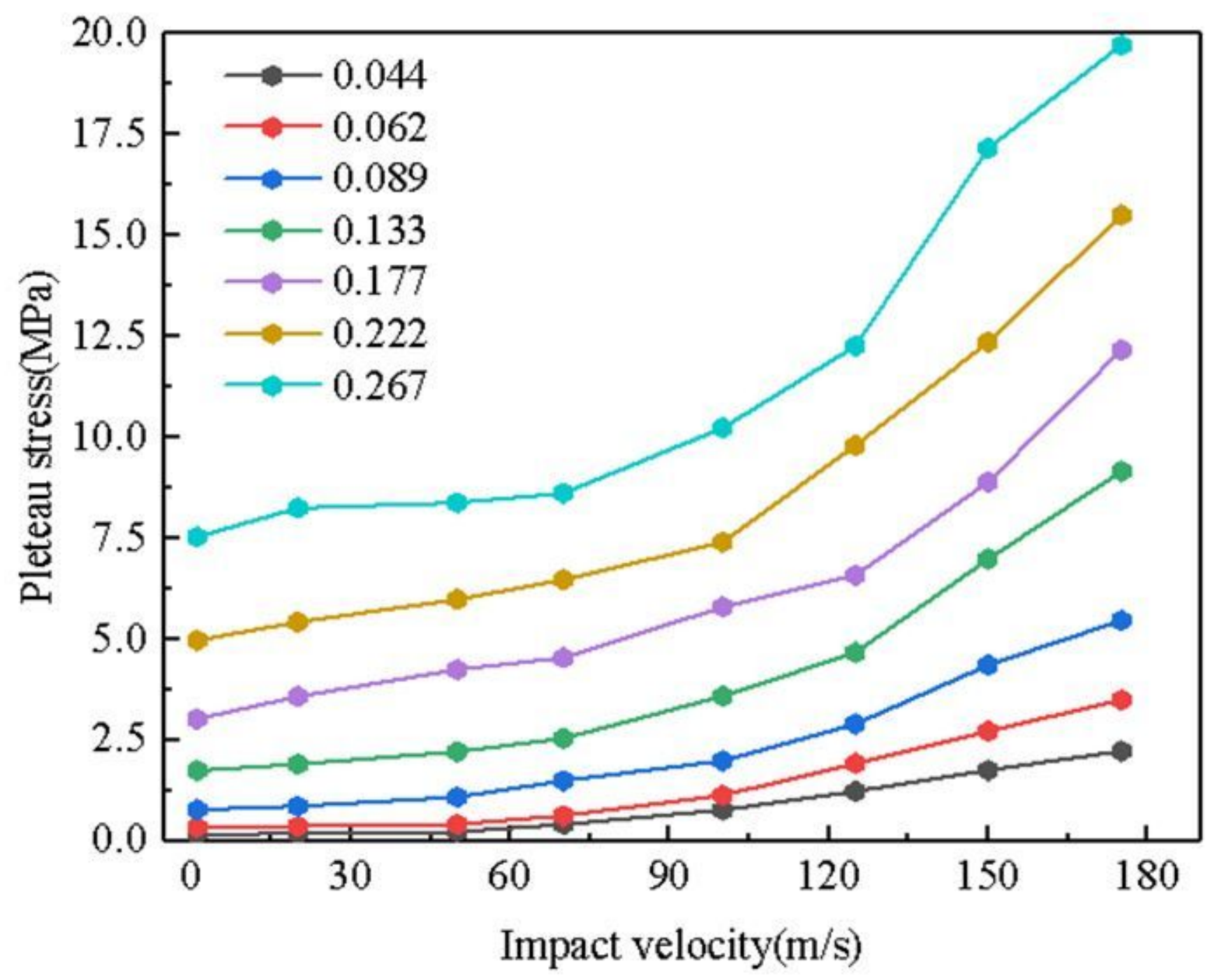

Figure 17

The plateau stress of $\mathrm{BRH}-\llbracket$ with different relative densities under different impact velocities. 


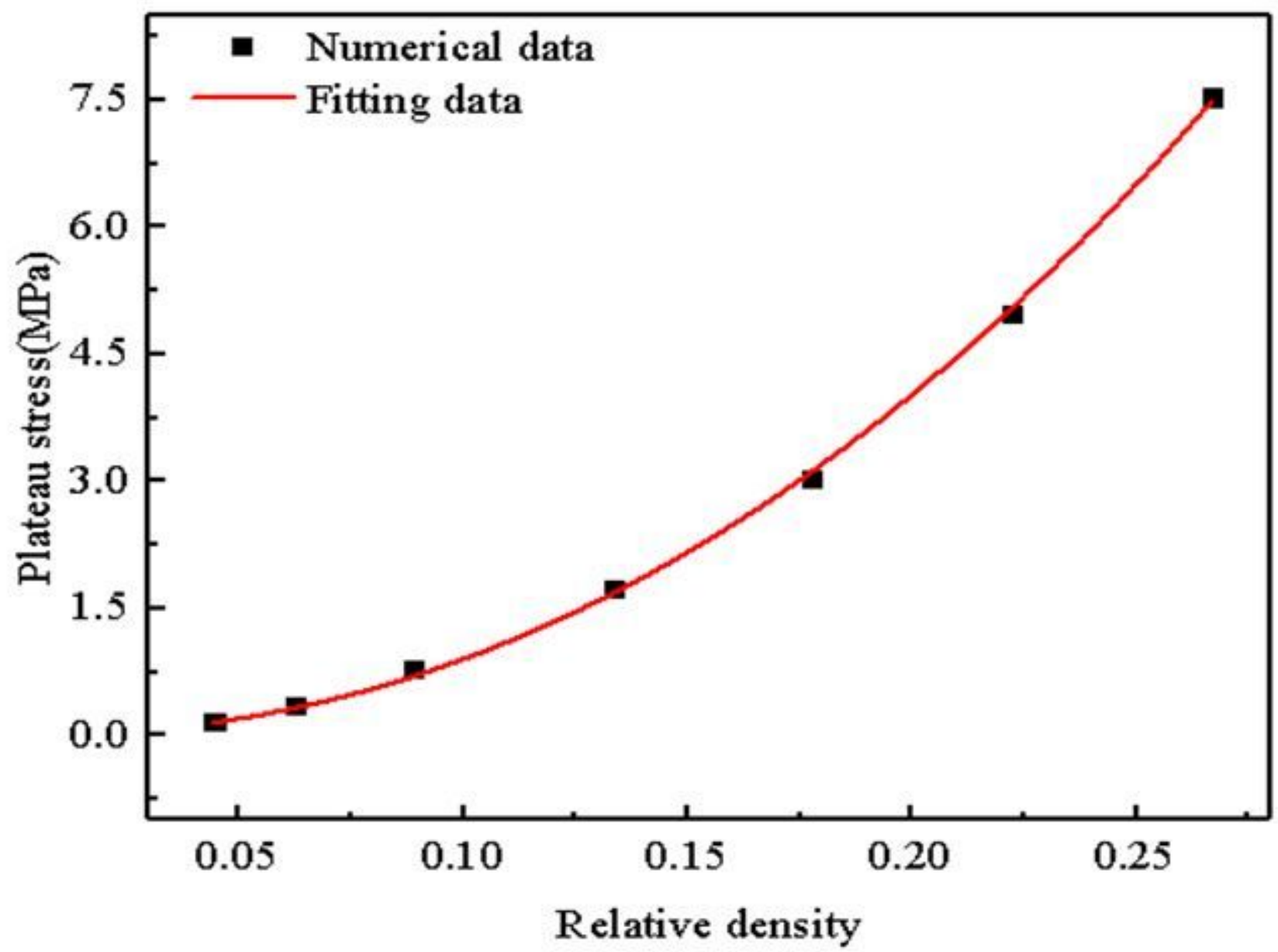

Figure 18

Quasi-static plateau stress comparisons between simulation results and fitting formula Eq. (8). 


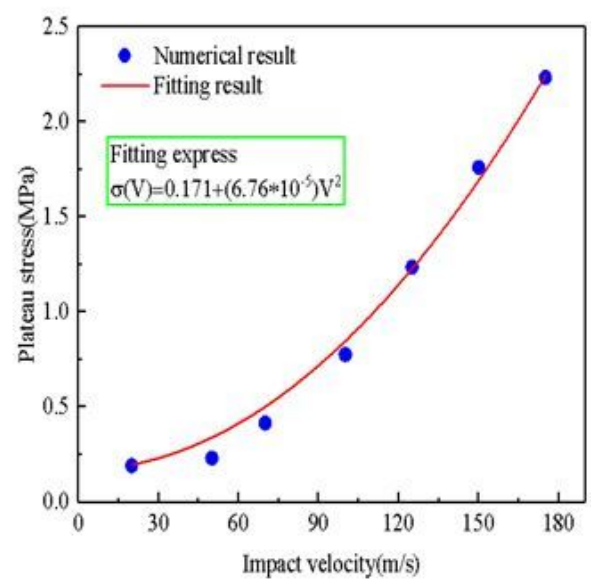

(a)

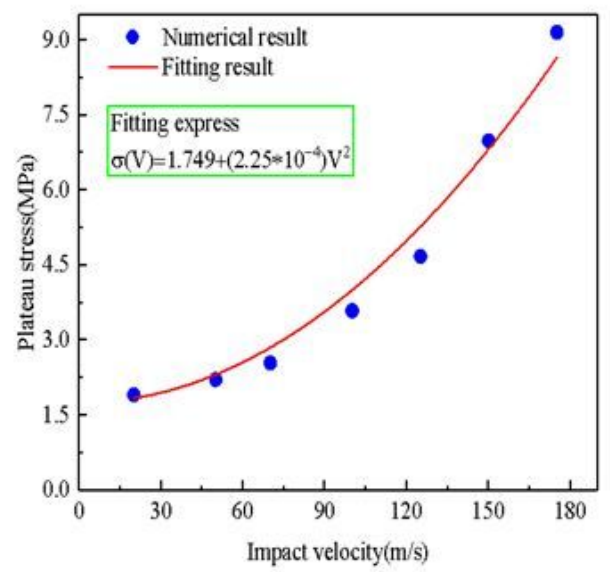

(d)

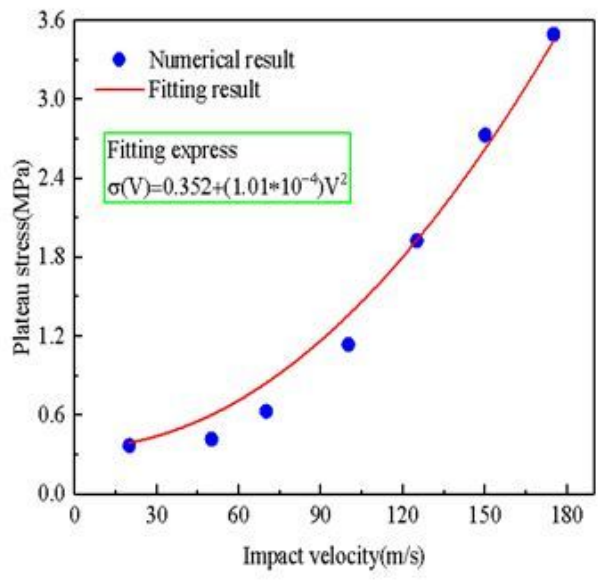

(b)

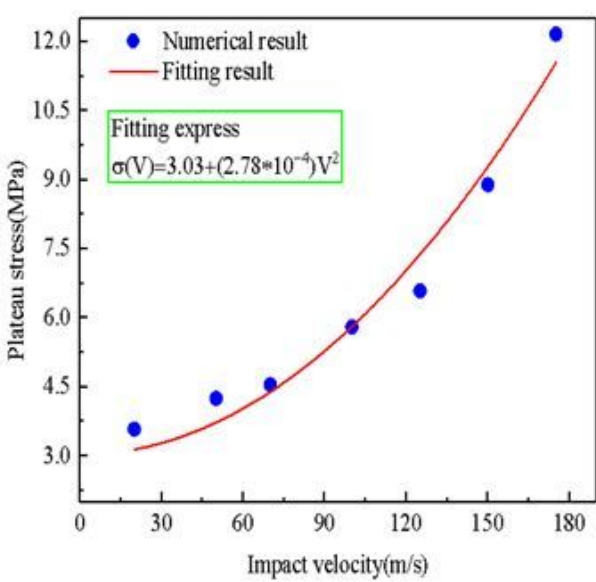

(e)

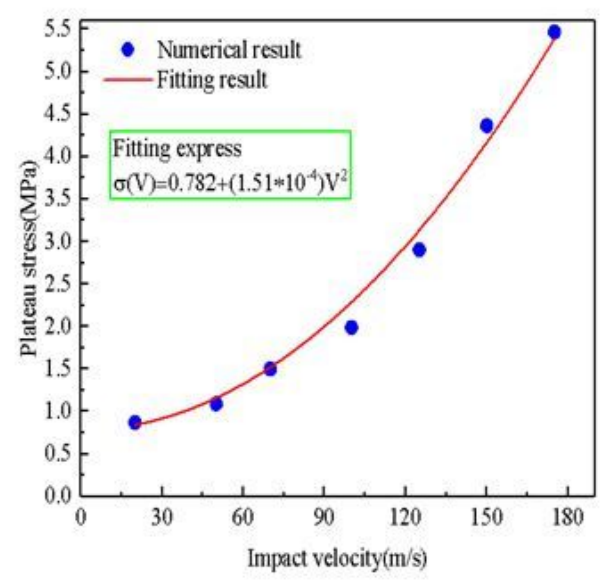

(c)

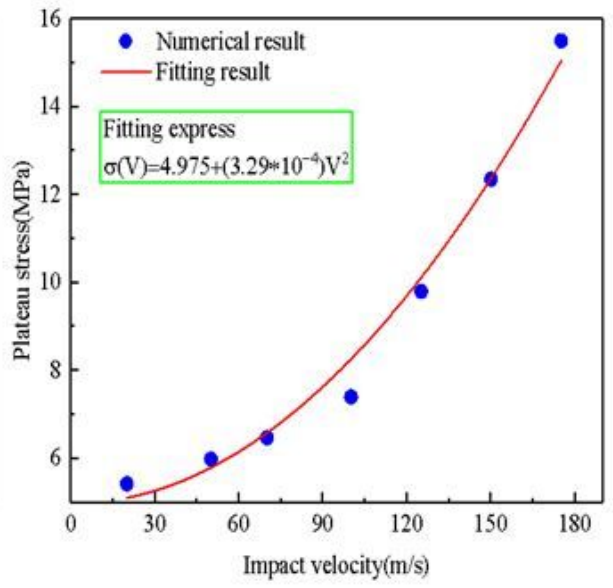

(f)

\section{Figure 19}

please see the manuscript file for the full caption

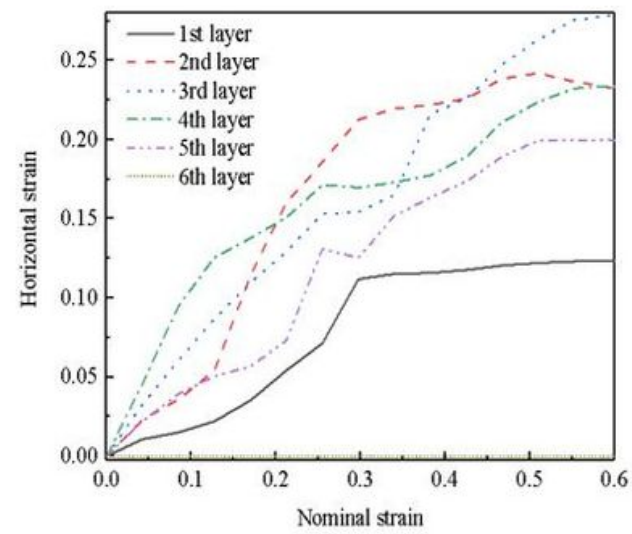

(a)

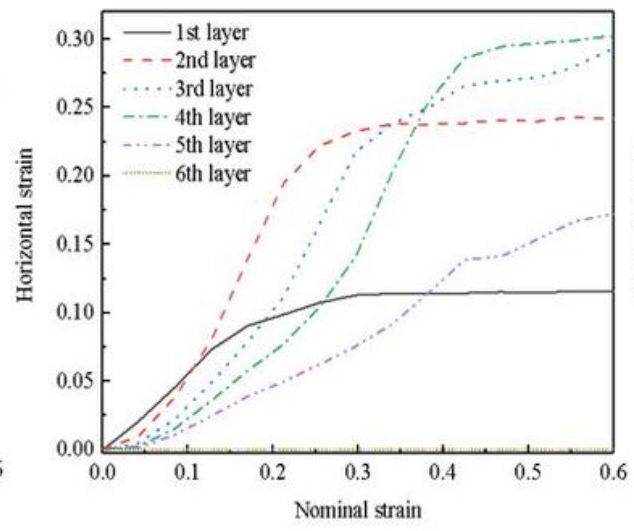

(b)

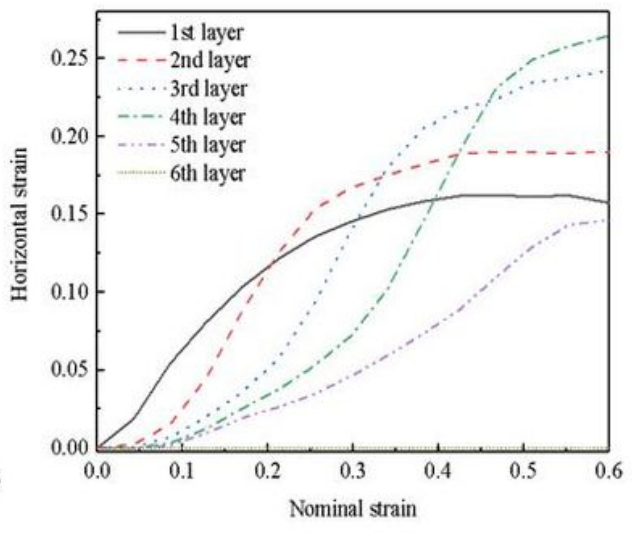

(c) 


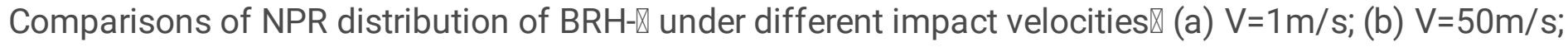
(c) $\mathrm{V}=100 \mathrm{~m} / \mathrm{s}$.

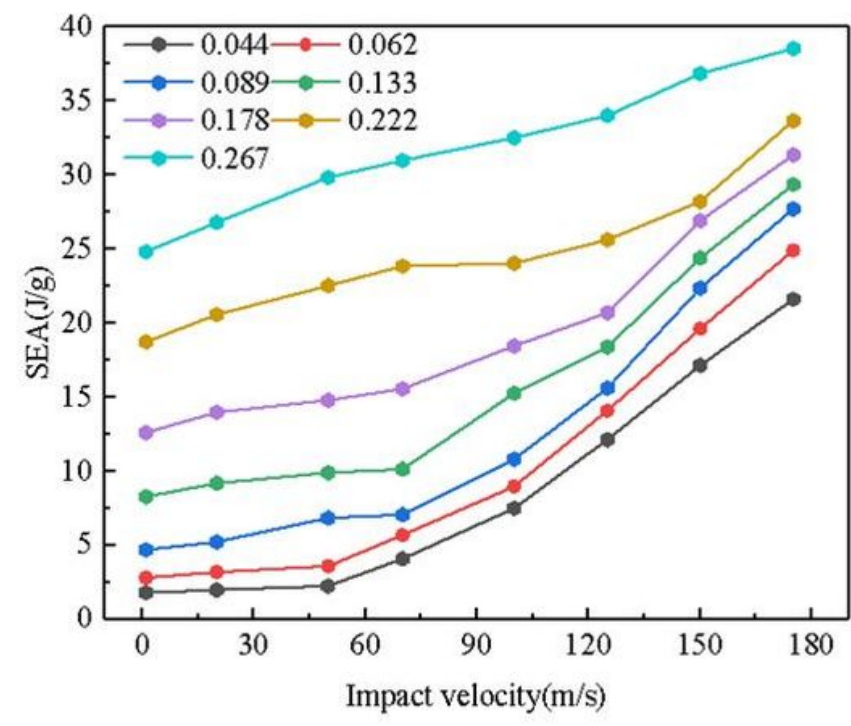

(a)

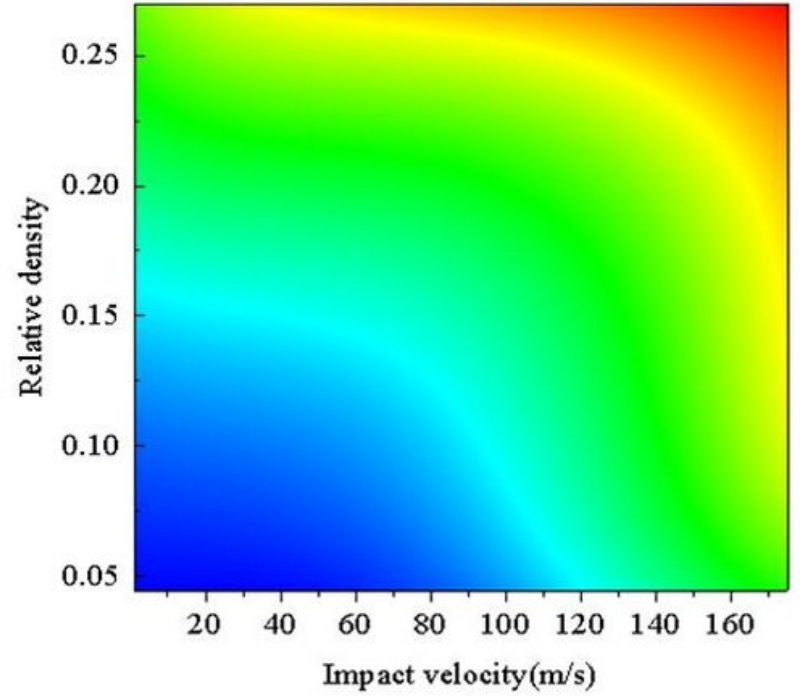

(b)

Figure 22

The influence of impact velocity and relative density on SEA of BRH-囚: (a) Dotted line diagram; (b) Contour diagram.

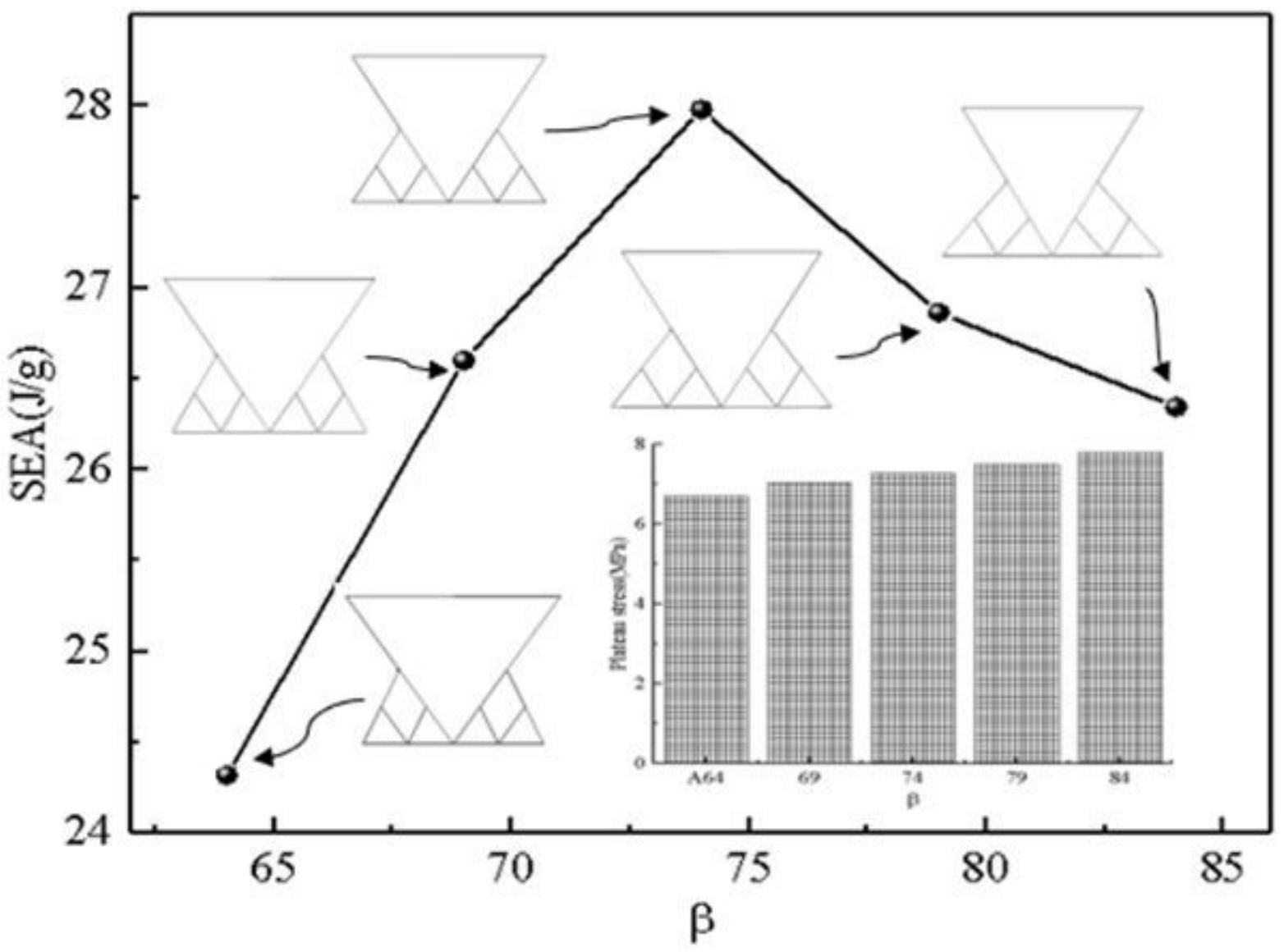




\section{Figure 23}

The influence of branch angle $\beta$ on SEA and plateau stress of BRH- $\square$.

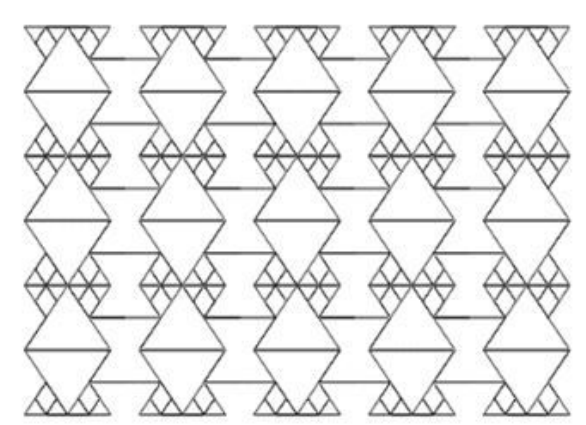

(a)

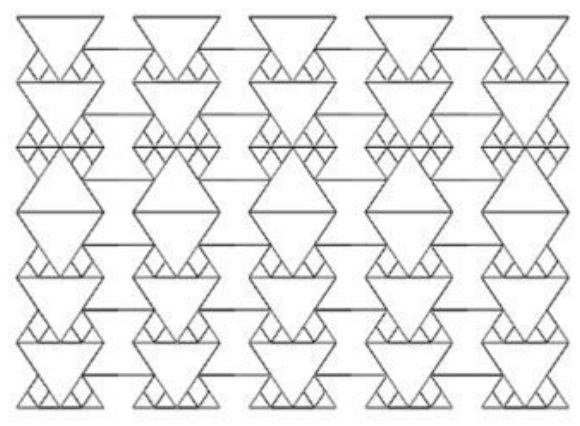

(b)

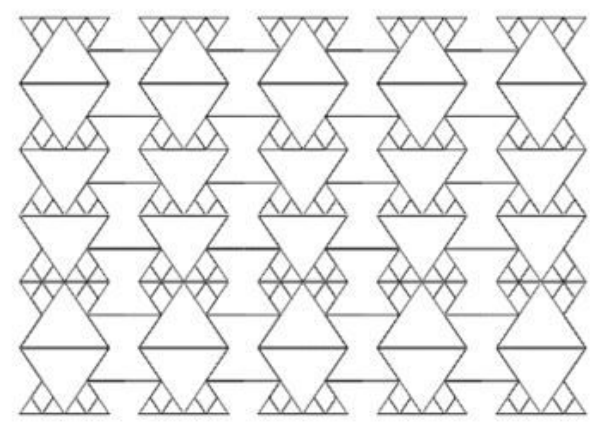

(c)

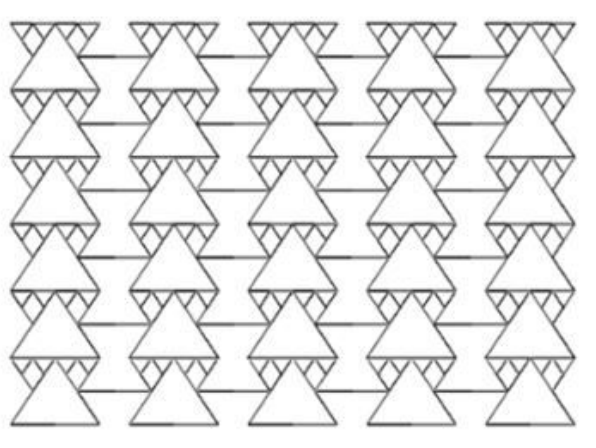

(d)

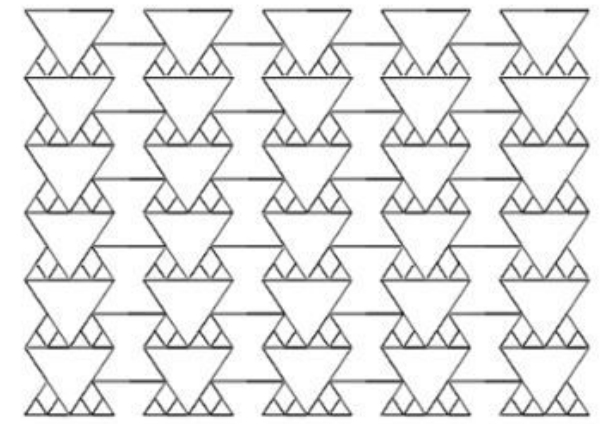

(e)

\section{Figure 24}

Different arrangements of BRH- $\rrbracket$. 


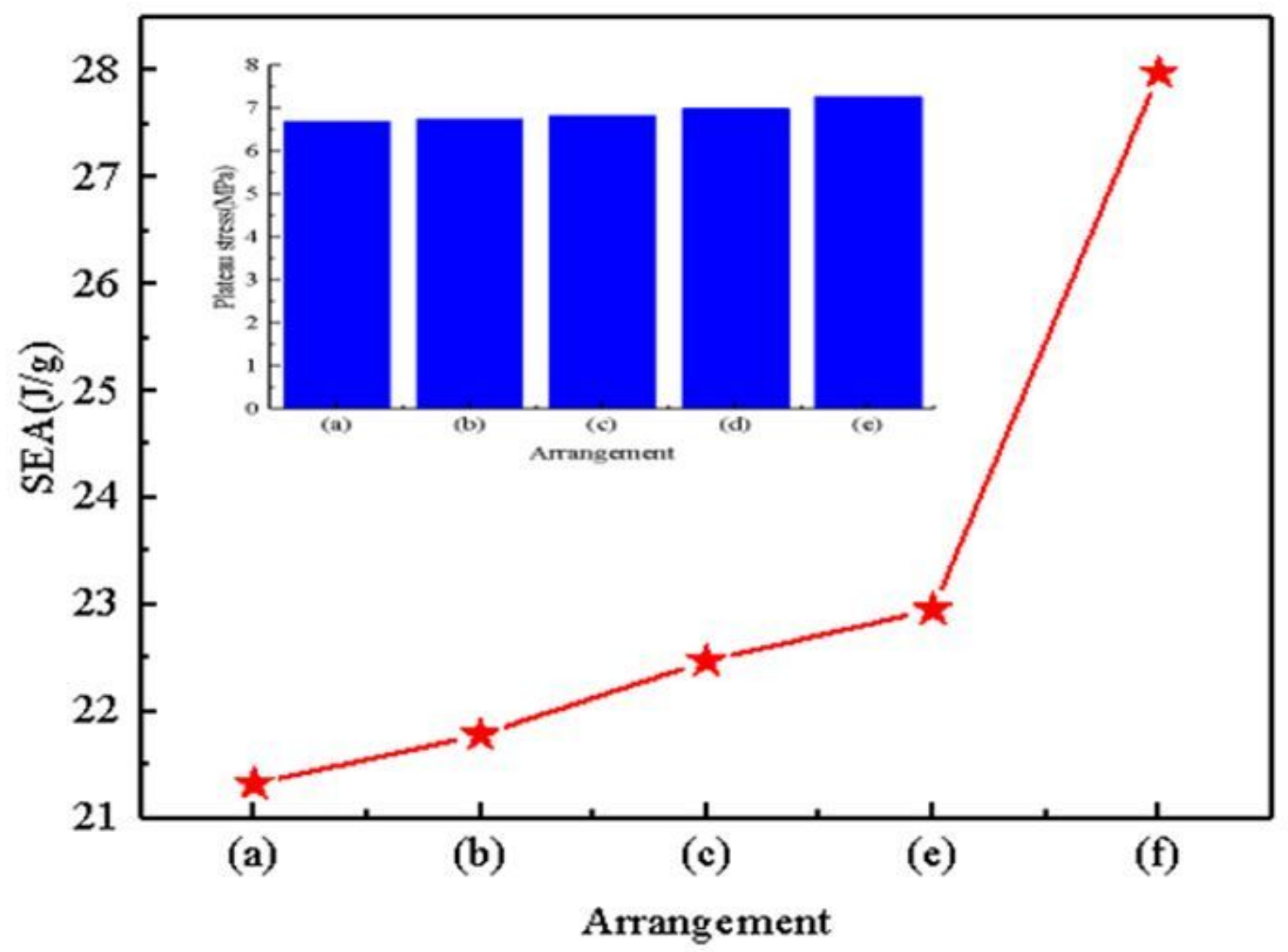

Figure 25

The influence of arrangements on SEA and plateau stress of BRH-囚.

\section{Supplementary Files}

This is a list of supplementary files associated with this preprint. Click to download.

- table.doc 